\title{
On the qualitative analysis of the fractional boundary value problem describing thermostat control model via $\psi$-Hilfer fractional operator
}

\author{
Chatthai Thaiprayoon', Weerawat Sudsutad ${ }^{2}$, Jehad Alzabut ${ }^{3}$, Sina Etemad ${ }^{4}$ and Shahram Rezapour ${ }^{4,5^{*}}$ (D)
}

\author{
"Correspondence: \\ sh.rezapour@azaruniv.ac.ir; \\ sh.rezapour@mail.cmuh.org.tw; \\ rezapourshahram@yahoo.ca \\ ${ }^{4}$ Department of Mathematics, \\ Azarbaijan Shahid Madani \\ University, Tabriz, Iran \\ ${ }^{5}$ Department of Medical Research, \\ China Medical University Hospital, \\ China Medical University, Taichung, \\ Taiwan \\ Full list of author information is \\ available at the end of the article
}

\begin{abstract}
In this research study, we are concerned with the existence and stability of solutions of a boundary value problem (BVP) of the fractional thermostat control model with $\psi$-Hilfer fractional operator. We verify the uniqueness criterion via the Banach fixed-point principle and establish the existence by using the Schaefer and Krasnoselskii fixed-point results. Moreover, we apply the arguments related to the nonlinear functional analysis to discuss various types of stability in the format of Ulam. Finally, by several examples we demonstrate applications of the main findings.
\end{abstract}

MSC: Primary 34A08; secondary 34A12; 39B10

Keywords: $\psi$-Hilfer operator; Existence of solutions; Fixed point; Ulam-Hyers stability; Uniqueness; Thermostat control model

\section{Springer}

\section{Introduction}

Fractional differential and integral equations have demonstrated high visibility and capability in applications of various topics related to physics, signal processing, mechanics, electromagnetics, economics, biology, and many more [1, 2]. Particularly speaking, it has been recognized that fractional integro-differential equations, whose kernels allow much freedom to describe various processes involving memory and hereditary properties, often appear in different fractional models caused by many real-life processes such as phenomena related to electromagnetic waves and heat transfer. Yang et al. [3] implemented a discussion on the steady heat-transfer in the context of fractal media by invoking the local fractional nonlinear integro-differential equations of Volterra type. Furthermore, electromagnetic waves in a wide range of dielectric media including the susceptibility following a fractional power law are formulated in the framework of integro-differential equations [4]. We can see some recent advances and applications of fractional modelings in several newly published researches such as [5-8]. Also, in some new papers, the advantages and power of mathematical modeling based on fractional operators are illustrated, and that is

(c) The Author(s) 2021. This article is licensed under a Creative Commons Attribution 4.0 International License, which permits use sharing, adaptation, distribution and reproduction in any medium or format, as long as you give appropriate credit to the original author(s) and the source, provide a link to the Creative Commons licence, and indicate if changes were made. The images or other third party material in this article are included in the article's Creative Commons licence, unless indicated otherwise in a credit line to the material. If material is not included in the article's Creative Commons licence and your intended use is not permitted by statutory regulation or exceeds the permitted use, you will need to obtain permission directly from the copyright holder. To view a copy of this licence, visit http://creativecommons.org/licenses/by/4.0/. 
why in recent years, many researchers prefer studying real processes and phenomena by applying newly defined versions of fractional operators (see, e.g., [9-18]).

Amongst important physio-electrical models, we are concerned with the thermostat control model. In this context a thermostat is a regulating instrument that measures the temperature of a given physical system and takes actions, provided that its temperature is maintained near a desired level. Thermostats are applied in any industrial system or controlling devices that cool or heat the temperature, examples including central heating, building heating, water heaters, air conditioners, water heaters, and also kitchen equipment such as refrigerators, ovens, and scientific and medical incubators. In [19] the authors demonstrated interest in the investigation of a thermostat control model insulated at $\varsigma=0$ with controller at $\varsigma=1$ and proposed the following boundary value problem (BVP) for the first time:

$$
\left\{\begin{array}{l}
v^{\prime \prime}(\varsigma)+h(\varsigma, v(\varsigma))=0, \quad \varsigma \in(0,1] \\
v^{\prime}(0)=0, \quad c v^{\prime}(1)+v(k)=0
\end{array}\right.
$$

in which $k \in(0,1)$, and $c>0$ is assumed to be an arbitrary parameter. Based on such a second-order mathematical model, the thermostat discharges or adds an amount of the heat with respect to the temperature detected by the existing sensor at $\varsigma=k$. They proved existence results for (1) by following the fixed point index theory in the context of integral equations of Hammerstein type.

Knowing the magnificent advantage of fractional derivatives, the authors in [20] studied the fractional-order thermostat control model

$$
\left\{\begin{array}{l}
{ }^{C} \mathfrak{D}^{\alpha} x(\varsigma)+f(\varsigma, x(\varsigma))=0, \quad \varsigma \in(0,1] \\
x^{\prime}(0)=0, \quad \lambda^{C} \mathfrak{D}^{\alpha-1} x(1)+x(\eta)=0,
\end{array}\right.
$$

where ${ }^{C} \mathfrak{D}^{\alpha}$ is the Caputo derivative of order $\alpha \in(1,2]$. Based on the hypothesis that the nonlinearity $f$ is assumed to be either superlinear or sublinear, the existence of positive solutions was proved by the help of the obtained Green's function and the Guo-Krasnoselskii fixed-point results. In their recent paper [21] the authors studied the fractional configuration of the thermostat control model subject to a convex-concave source term. They used the fixed point technique to prove the existence and uniqueness of positive solutions and provided an iterative scheme to approximate the obtained solutions. For more details on fractional thermostat control models, the reader can consult [22-26]. Exploring this literature, we can notice that the reported results were restricted to the existence of positive solutions and their properties. However, to the best of the authors' knowledge, no results were observed on the thermostat control model in the frame of generalized fractional operators. Further, the stability of solutions of fractional thermostat control models was not addressed yet.

Motivated by the above discussions, we consider a category of $\psi$-Hilfer nonlinear implicit fractional boundary value problems (FBVPs) describing the thermostat control model of the form

$$
\left\{\begin{array}{l}
{ }^{H} \mathfrak{D}_{0^{+}}^{\alpha, \rho ; \psi} x(\varsigma)=f\left(\varsigma, x(\theta \varsigma), \mathcal{I}_{0^{+}}^{q ; \psi} x(\varepsilon \varsigma)\right), \quad \varsigma \in(0, T], \\
\sum_{i=1}^{m} \omega_{i}^{H} \mathfrak{D}_{0^{+}}^{\beta_{i}, \rho ; \psi} x\left(\xi_{i}\right)=A, \quad \sum_{j=1}^{n} \lambda_{j}{ }^{H} \mathfrak{D}_{0^{+}}^{\mu_{j}, \rho ; \psi} x\left(\sigma_{j}\right)+\sum_{k=1}^{r} \delta_{k} x\left(\eta_{k}\right)=B,
\end{array}\right.
$$


where ${ }^{H} \mathfrak{D}_{0^{+}}^{v, \rho ; \psi}$ denotes the $\psi$-Hilfer derivative operators of order $v=\left\{\alpha, \beta_{i}, \mu_{j}\right\}, \alpha \in(1,2]$, $\beta_{i}, \mu_{j} \in(0,1], A, B, \omega_{i}, \lambda_{j}, \delta_{k} \in \mathbb{R}, \xi_{i}, \sigma_{j}, \eta_{k} \in(0, T), i=1,2, \ldots, m, j=1,2, \ldots, n, k=1, \ldots, r$, $\rho \in[0,1], \mathcal{I}_{0^{+}}^{q ; \psi}$ is the $\psi$-RL-integral of order $q>0, \theta, \varepsilon \in(0,1], f \in \mathcal{C}\left(\mathbb{J} \times \mathbb{R}^{2}, \mathbb{R}\right)$, and $\mathbb{J}:=$ $[0, T]$ with $T>0$. We establish existence and stability results for (3). We employ fixed point hypotheses to prove the existence results and we use the techniques of nonlinear functional analysis to study the stability in the Ulam sense. We present our results in a general platform, which covers many particular cases for specific values of $\rho$ and $\psi$. For some relevant results, we refer the reader to recent papers [27-33].

The remaining parts of the research study adhere to the following plan. In Sect. 2, we define the norms, spaces, and other essential notions and lemmas related to the $\psi$-Hilfer fractional operator. Further, we derive the equivalent integral representation associated with the linear problem and state some fixed point theorems. We present the existence and uniqueness results in terms of three different fixed-point criteria in Sect. 3. In Sect. 4, we systematically present stabilization analysis of problem (3). In Sect. 5, we construct three particular examples, where the validity of the proposed results is verified. We terminate the investigation by conclusions.

\section{Primitive notions}

In this section, we give important basic definitions and primitive concepts of fractional calculus, which are useful throughout this paper.

We denote by $\mathbb{E}=\mathcal{C}(\mathbb{J}, \mathbb{R})$ the Banach space of continuous mappings on $\mathbb{I}$ with supnorm $\|x\|=\sup _{\varsigma \in \mathbb{J}}\{|x(\varsigma)|\}$ for $x \in \mathbb{E}$.

We also define the space of $n$ times absolutely continuous functions

$$
\mathcal{A C}^{n}[\mathbb{J}, \mathbb{R}]=\left\{f: \mathbb{J} \rightarrow \mathbb{R} ; f^{(n-1)} \in \mathcal{A C}[\mathbb{J}, \mathbb{R}]\right\} .
$$

Definition 2.1 ([34]) Let $\psi(\varsigma) \in \mathcal{C}^{1}([a, b], \mathbb{R})$ be an increasing function with $\psi^{\prime}(\varsigma) \neq 0$ for each $\varsigma \in[a, b]$. The $\alpha$ th- $\psi$-RL-fractional integral of $f$ depending on the function $\psi$ is defined as

$$
\mathcal{I}_{a^{+}}^{\alpha ; \psi} f(\varsigma)=\frac{1}{\Gamma(\alpha)} \int_{a}^{\varsigma} \psi^{\prime}(s)(\psi(\varsigma)-\psi(s))^{\alpha-1} f(s) d s, \quad \varsigma>a>0, \alpha>0,
$$

where $\Gamma$ is the gamma function.

Definition 2.2 ([34]) Let $\psi$ be as above with $\psi^{\prime}(\varsigma) \neq 0$. The $\alpha$ th- $\psi$-RL-fractional derivative of $f$ depending on $\psi$ is defined as

$$
\begin{aligned}
\mathfrak{D}_{a^{+}}^{\alpha ; \psi} f(\varsigma) & =\left(\frac{1}{\psi^{\prime}(\varsigma)} \frac{d}{d \varsigma}\right)^{n} \mathcal{I}_{a^{+}}^{n-\alpha ; \psi} f(\varsigma) \\
& =\frac{1}{\Gamma(n-\alpha)}\left(\frac{1}{\psi^{\prime}(\varsigma)} \frac{d}{d \varsigma}\right)^{n} \int_{a}^{\varsigma} \psi^{\prime}(s)(\psi(\varsigma)-\psi(s))^{n-\alpha-1} f(s) d s, \quad \alpha>0,
\end{aligned}
$$

where $n=[\alpha]+1$.

Definition 2.3 ([35]) Let $\gamma=\alpha+\rho(n-\alpha), \alpha \in(n-1, n), f \in \mathcal{C}^{n}([a, b], \mathbb{R})$, and let $\psi(\varsigma) \in$ $\mathcal{C}^{1}([a, b], \mathbb{R})$ be increasing with $\psi^{\prime}(\varsigma) \neq 0$ for each $\varsigma \in[a, b]$. Then the $\alpha$ th- $\psi$-Hilfer deriva- 
tive of $f$ of type $\rho \in[0,1]$ depending on the function $\psi$ is given by

$$
{ }^{H} \mathfrak{D}_{a^{+}}^{\alpha, \rho ; \psi} f(\varsigma)=\mathcal{I}_{a^{+}}^{\rho(n-\alpha) ; \psi}\left(\frac{1}{\psi^{\prime}(\varsigma)} \frac{d}{d \varsigma}\right)^{n} \mathcal{I}_{a^{+}}^{(1-\rho)(n-\alpha) ; \psi} f(\varsigma)=\mathcal{I}_{a^{+}}^{\gamma-\alpha ; \psi} \mathfrak{D}_{a^{+}}^{\gamma ; \psi} f(\varsigma),
$$

where $\mathfrak{D}_{a^{+}}^{\gamma ; \psi} f(\varsigma)=\mathfrak{D}_{a^{+}}^{n ; \psi} \mathcal{I}_{a^{+}}^{(1-\rho)(n-\alpha) ; \psi} f(\varsigma)$.

Lemma 2.4 ([34]) Let $\alpha, \beta>0$. In this case, we have the $\psi$-semigroup property

$$
\mathcal{I}_{a^{+}}^{\alpha ; \psi} \mathcal{I}_{a^{+}}^{\beta ; \psi} f(\varsigma)=\mathcal{I}_{a^{+}}^{\alpha+\beta ; \psi} f(\varsigma), \quad \varsigma>a
$$

Next, we present the following properties.

Proposition 2.5 $([34,35])$ Let $\varsigma>$ a and consider $\chi^{v}(\varsigma)=(\psi(\varsigma)-\psi(a))^{v-1}$. Then, for $v>0$ and $\alpha \geq 0$, we have the following properties:

(i) $\mathcal{I}_{a^{+}}^{\alpha ; \psi} \chi^{v}(\varsigma)=\frac{\Gamma(v)}{\Gamma(v+\alpha)} \chi^{v+\alpha}(\varsigma)$;

(ii) $\mathfrak{D}_{a^{+}}^{\alpha, \rho ; \psi} \chi^{v}(\varsigma)=\frac{\Gamma(v)}{\Gamma(v-\alpha)} \chi^{v-\alpha}(\varsigma)$;

(iii) ${ }^{H} \mathfrak{D}_{a^{+}}^{\alpha, \rho ; \psi} \chi^{v}(\varsigma)=\frac{\Gamma(v)}{\Gamma(v-\alpha)} \chi^{v}(\varsigma), v>\gamma=\alpha+\rho(2-\alpha)$.

Lemma 2.6 Let $\alpha \in(m-1, m), \beta \in(n-1, n), n, m \in \mathbb{N}, n \leq m, \rho \in[0,1]$, and $\alpha>\beta+\rho(n-$ $\beta)$. If $h \in \mathcal{C}_{1-\gamma, \psi}(\mathbb{J}, \mathbb{R})$, then

$$
{ }^{H} \mathfrak{D}_{a^{+}}^{\beta, \rho ; \psi} \mathcal{I}_{a^{+}}^{\alpha ; \psi} h(\varsigma)=\mathcal{I}_{0^{+}}^{\alpha-\beta ; \psi} h(\varsigma) .
$$

Proof Letting $\xi=\beta+\rho(n-\beta)$ with $n-1<\xi<n$, we get

$$
\begin{aligned}
& { }^{H} \mathfrak{D}_{a^{+}}^{\beta, \rho ; \psi}\left(\mathcal{I}_{a^{+}}^{\alpha ; \psi} h(\varsigma)\right)=\mathcal{I}_{a^{+}}^{\xi-\beta ; \psi} \mathfrak{D}_{a^{+}}^{\xi ; \psi}\left(\mathcal{I}_{a^{+}}^{\alpha ; \psi} h(\varsigma)\right) \\
& =\mathcal{I}_{a^{+}}^{\xi-\beta ; \psi}\left(\frac{1}{\psi^{\prime}(\varsigma)} \frac{d}{d \varsigma}\right)^{n} \mathcal{I}_{a^{+}}^{n-\xi ; \psi}\left(\mathcal{I}_{a^{+}}^{\alpha ; \psi} h(\varsigma)\right) \\
& =\mathcal{I}_{a^{+}}^{\xi-\beta ; \psi}\left(\frac{1}{\psi^{\prime}(\varsigma)} \frac{d}{d \varsigma}\right)^{n} \mathcal{I}_{a^{+}}^{n-\xi+\alpha ; \psi} h(\varsigma) .
\end{aligned}
$$

By Definition 2.1 we obtain

$$
\begin{aligned}
\left(\frac{1}{\psi^{\prime}(\varsigma)} \frac{d}{d \varsigma}\right) \mathcal{I}_{0^{+}}^{n-\xi+\alpha ; \psi} h(\varsigma) \\
=\frac{1}{\psi^{\prime}(\varsigma)} \frac{d}{d \varsigma}\left(\frac{1}{\Gamma(n-\xi+\alpha)} \int_{a}^{\varsigma} \psi^{\prime}(s)(\psi(\varsigma)-\psi(s))^{n+\alpha-\xi-1} h(s) d s\right) \\
=\frac{1}{\Gamma(n-\xi+\alpha)} \frac{1}{\psi^{\prime}(\varsigma)} \\
\quad \times\left(\int_{a}^{\varsigma}(n+\alpha-\xi-1) \psi^{\prime}(s) \psi^{\prime}(\varsigma)(\psi(\varsigma)-\psi(s))^{n+\alpha-\xi-2} h(s) d s\right) \\
=\frac{1}{\Gamma(n-\xi+\alpha-1)} \int_{a}^{\varsigma} \psi^{\prime}(s)(\psi(\varsigma)-\psi(s))^{n+\alpha-\xi-2} h(s) d s \\
=\mathcal{I}_{a^{+}}^{n-\xi+\alpha-1 ; \psi} h(\varsigma)
\end{aligned}
$$


and

$$
\begin{aligned}
\left(\frac{1}{\psi^{\prime}(\varsigma)} \frac{d}{d \varsigma}\right)^{2} \mathcal{I}_{0^{+}}^{n-\xi+\alpha ; \psi} h(\varsigma) \\
=\frac{1}{\psi^{\prime}(\varsigma)} \frac{d}{d \varsigma}\left(\frac{1}{\Gamma(n-\xi+\alpha-1)} \int_{a}^{\varsigma} \psi^{\prime}(s)(\psi(\varsigma)-\psi(s))^{n+\alpha-\xi-2} h(s) d s\right) \\
=\frac{1}{\Gamma(n-\xi+\alpha-1)} \frac{1}{\psi^{\prime}(\varsigma)} \\
\quad \times\left(\int_{a}^{\varsigma}(n+\alpha-\xi-2) \psi^{\prime}(s) \psi^{\prime}(\varsigma)(\psi(\varsigma)-\psi(s))^{n+\alpha-\xi-3} h(s) d s\right) \\
=\frac{1}{\Gamma(n-\xi+\alpha-2)} \int_{a}^{\varsigma} \psi^{\prime}(s)(\psi(\varsigma)-\psi(s))^{n+\alpha-\xi-3} h(s) d s \\
=\mathcal{I}_{a^{+}}^{n-\xi+\alpha-2 ; \psi} h(\varsigma) .
\end{aligned}
$$

Repeating the above process, we have

$$
\begin{aligned}
& \left(\frac{1}{\psi^{\prime}(\varsigma)} \frac{d}{d \varsigma}\right)^{n} \mathcal{I}_{0^{+}}^{n-\xi+\alpha ; \psi} h(\varsigma) \\
& \quad=\frac{1}{\psi^{\prime}(\varsigma)} \frac{d}{d \varsigma}\left(\frac{1}{\Gamma(\alpha-\xi)} \int_{a}^{\varsigma} \psi^{\prime}(s)(\psi(\varsigma)-\psi(s))^{\alpha-\xi-1} h(s) d s\right) \\
& \quad=\frac{1}{\Gamma(\alpha-\xi+1)} \frac{1}{\psi^{\prime}(\varsigma)}\left(\int_{a}^{\varsigma}(\alpha-\xi) \psi^{\prime}(s) \psi^{\prime}(\varsigma)(\psi(\varsigma)-\psi(s))^{\alpha-\xi-1} h(s) d x\right) \\
& =\frac{1}{\Gamma(\xi+\alpha)} \int_{a}^{\varsigma} \psi^{\prime}(s)(\psi(\varsigma)-\psi(s))^{\alpha-\xi-1} h(s) d s \\
& =\mathcal{I}_{a^{+}}^{\alpha-\xi ; \psi} h(\varsigma),
\end{aligned}
$$

which implies that

$$
{ }^{H} \mathfrak{D}_{a^{+}}^{\beta, \rho ; \psi}\left(\mathcal{I}_{a^{+}}^{\alpha ; \psi} h(\varsigma)\right)=\mathcal{I}_{a^{+}}^{\xi-\beta ; \psi} \mathcal{I}_{a^{+}}^{\alpha-\xi ; \psi} h(\varsigma)=\mathcal{I}_{a^{+}}^{\alpha-\beta ; \psi} h(\varsigma)
$$

This completes the proof.

Lemma 2.7 ([35]) Let $f \in \mathcal{C}^{n}(\mathbb{J}, \mathbb{R}), \gamma=\alpha+\rho(n-\alpha)$ with $\alpha \in(n-1, n)$, and $\rho \in[0,1]$. Then

$$
\mathcal{I}_{a^{+}}^{\alpha ; \psi H} \mathfrak{D}_{a^{+}}^{\alpha, \rho ; \psi} f(\varsigma)=f(\varsigma)-\sum_{k=1}^{n} \frac{(\psi(\varsigma)-\psi(a))^{\gamma-k}}{\Gamma(\gamma-k+1)} f_{\psi}^{[n-k]} \mathcal{I}_{a^{+}}^{(1-\rho)(n-\alpha) ; \psi} f(a)
$$

for any $\varsigma \in \mathbb{J}$, so that $f_{\psi}^{[n]} f(\varsigma):=\left(\frac{1}{\psi^{\prime}(\varsigma)} \frac{d}{d \varsigma}\right)^{n} f(\varsigma)$.

Lemma 2.8 Let $\Omega \neq 0, \alpha \in(1,2], \beta_{1}, \ldots, \beta_{m}, \mu_{1}, \ldots, \mu_{n} \in(0,1], \rho \in[0,1]$, and $\gamma=\alpha+\rho(2-$ $\alpha$ ). Suppose that $h \in \mathbb{E}$. Then $x \in \mathcal{C}^{2}(\mathbb{J}, \mathbb{R})$ is a solution of

$$
\left\{\begin{array}{l}
{ }^{H} \mathfrak{D}_{0^{+}}^{\alpha, \rho ; \psi} x(\varsigma)=h(\varsigma), \quad \varsigma \in(0, T], \\
\sum_{i=1}^{m} \omega_{i}^{H} \mathfrak{D}_{0^{+}}^{\beta_{i}, \rho ; \psi} x\left(\xi_{i}\right)=A, \quad \sum_{j=1}^{n} \lambda_{j}^{H} \mathfrak{D}_{0^{+}}^{\mu_{j}, \rho ; \psi} x\left(\sigma_{j}\right)+\sum_{k=1}^{r} \delta_{k} x\left(\eta_{k}\right)=B,
\end{array}\right.
$$


iff $x$ fulfills the $\psi$-integral equation

$$
\begin{aligned}
x(\varsigma)= & \mathcal{I}_{0^{+}}^{\alpha ; \psi} h(\varsigma)+\frac{(\psi(\varsigma)-\psi(0))^{\gamma-1}}{\Omega \Gamma(\gamma)}\left[\Omega_{4}\left(A-\sum_{i=1}^{m} \omega_{i} \mathcal{I}_{0^{+}}^{\alpha-\beta_{i} ; \psi} h\left(\xi_{i}\right)\right)\right. \\
& \left.-\Omega_{2}\left(B-\sum_{j=1}^{n} \lambda_{j} \mathcal{I}_{0^{+}}^{\alpha-\mu_{j} ; \psi} h\left(\sigma_{j}\right)-\sum_{k=1}^{r} \delta_{k} \mathcal{I}_{0^{+}}^{\alpha ; \psi} h\left(\eta_{k}\right)\right)\right] \\
& +\frac{(\psi(\varsigma)-\psi(0))^{\gamma-2}}{\Omega \Gamma(\gamma-1)}\left[\Omega_{1}\left(B-\sum_{j=1}^{n} \lambda_{j} \mathcal{I}_{0^{+}}^{\alpha-\mu_{j} ; \psi} h\left(\sigma_{j}\right)-\sum_{k=1}^{r} \delta_{k} \mathcal{I}_{0^{+}}^{\alpha ; \psi} h\left(\eta_{k}\right)\right)\right. \\
& \left.-\Omega_{3}\left(A-\sum_{i=1}^{m} \omega_{i} \mathcal{I}_{0^{+}}^{\alpha-\beta_{i} ; \psi} h\left(\xi_{i}\right)\right)\right],
\end{aligned}
$$

where

$$
\begin{aligned}
& \Omega_{1}=\sum_{i=1}^{m} \frac{\omega_{i}\left(\psi\left(\xi_{i}\right)-\psi(0)\right)^{\gamma-\beta_{i}-1}}{\Gamma\left(\gamma-\beta_{i}\right)}, \\
& \Omega_{2}=\sum_{i=1}^{m} \frac{\omega_{i}\left(\psi\left(\xi_{i}\right)-\psi(0)\right)^{\gamma-\beta_{i}-2}}{\Gamma\left(\gamma-\beta_{i}-1\right)}, \\
& \Omega_{3}=\sum_{j=1}^{n} \frac{\lambda_{j}\left(\psi\left(\sigma_{j}\right)-\psi(0)\right)^{\gamma-\mu_{j}-1}}{\Gamma\left(\gamma-\mu_{j}\right)}+\sum_{k=1}^{r} \frac{\delta_{k}\left(\psi\left(\eta_{k}\right)-\psi(0)\right)^{\gamma-1}}{\Gamma(\gamma)}, \\
& \Omega_{4}=\sum_{j=1}^{n} \frac{\lambda_{j}\left(\psi\left(\sigma_{j}\right)-\psi(0)\right)^{\gamma-\mu_{j}-2}}{\Gamma\left(\gamma-\mu_{j}-1\right)}+\sum_{k=1}^{r} \frac{\delta_{k}\left(\psi\left(\eta_{k}\right)-\psi(0)\right)^{\gamma-2}}{\Gamma(\gamma-1)}, \\
& \Omega=\Omega_{1} \Omega_{4}-\Omega_{2} \Omega_{3} .
\end{aligned}
$$

Proof Let $x \in \mathbb{E}$ be a solution of problem (4). Taking the operator $\mathcal{I}_{0^{+}}^{\alpha ; \psi}$ on both sides of (4) and using Lemma 2.7, we have

$$
x(\varsigma)=\mathcal{I}_{0^{+}}^{\alpha ; \psi} h(\varsigma)+\frac{(\psi(\varsigma)-\psi(0))^{\gamma-1}}{\Gamma(\gamma)} c_{1}+\frac{(\psi(\varsigma)-\psi(0))^{\gamma-2}}{\Gamma(\gamma-1)} c_{2},
$$

where $c_{1}, c_{2} \in \mathbb{R}$ are arbitrary real constants.

Taking the operators ${ }^{H} \mathfrak{D}_{0^{+}}^{\beta_{i}, \rho ; \psi}$ and ${ }^{H} \mathfrak{D}_{0^{+}}^{\mu_{j}, \rho ; \psi}$ into (11), we obtain

$$
\begin{aligned}
& { }^{H} \mathfrak{D}_{0^{+}}^{\beta_{j}, \rho ; \psi} x(\varsigma)=\mathcal{I}_{0^{+}}^{\alpha-\beta_{i} ; \psi} h(\varsigma)+\frac{(\psi(\varsigma)-\psi(0))^{\gamma-\beta_{i}-1}}{\Gamma\left(\gamma-\beta_{i}\right)} c_{1}+\frac{(\psi(\varsigma)-\psi(0))^{\gamma-\beta_{i}-2}}{\Gamma\left(\gamma-\beta_{i}-1\right)} c_{2}, \\
& { }^{H} \mathfrak{D}_{0^{+}}^{\mu_{j}, \rho ; \psi} x(\varsigma)=\mathcal{I}_{0^{+}}^{\alpha-\mu_{j} ; \psi} h(\varsigma)+\frac{(\psi(\varsigma)-\psi(0))^{\gamma-\mu_{j}-1}}{\Gamma\left(\gamma-\mu_{j}\right)} c_{1}+\frac{(\psi(\varsigma)-\psi(0))^{\gamma-\mu_{j}-2}}{\Gamma\left(\gamma-\mu_{j}-1\right)} c_{2} .
\end{aligned}
$$

From the first and second boundary conditions in (3) we get the system

$$
\left\{\begin{array}{l}
\Omega_{1} c_{1}+\Omega_{2} c_{2}=A-\sum_{i=1}^{m} \omega_{i} \mathcal{I}_{0^{+}}^{\alpha-\beta_{i} ; \psi} h\left(\xi_{i}\right), \\
\Omega_{3} c_{1}+\Omega_{4} c_{2}=B-\sum_{j=1}^{n} \lambda_{j} \mathcal{I}_{0^{+}}^{\alpha-\mu_{j} ; \psi} h\left(\sigma_{j}\right)-\sum_{k=1}^{r} \delta_{k} \mathcal{I}_{0^{+}}^{\alpha ; \psi} h\left(\eta_{k}\right),
\end{array}\right.
$$


where $\Omega_{1}, \Omega_{2}, \Omega_{3}$, and $\Omega_{4}$ are given by (6), (7), (8), and (9), respectively. Solving the system, it follows that

$$
\begin{aligned}
c_{1}= & \frac{1}{\Omega}\left[\Omega_{4}\left(A-\sum_{i=1}^{m} \omega_{i} \mathcal{I}_{0^{+}}^{\alpha-\beta_{i} ; \psi} h\left(\xi_{i}\right)\right)\right. \\
& \left.-\Omega_{2}\left(B-\sum_{j=1}^{n} \lambda_{j} \mathcal{I}_{0^{+}}^{\alpha-\mu_{j} ; \psi} h\left(\sigma_{j}\right)-\sum_{k=1}^{r} \delta_{k} \mathcal{I}_{0^{+}}^{\alpha ; \psi} h\left(\eta_{k}\right)\right)\right], \\
c_{2}= & \frac{1}{\Omega}\left[\Omega_{1}\left(B-\sum_{j=1}^{n} \lambda_{j} \mathcal{I}_{0^{+}}^{\alpha-\mu_{j} ; \psi} h\left(\sigma_{j}\right)-\sum_{k=1}^{r} \delta_{k} \mathcal{I}_{0^{+}}^{\alpha ; \psi} h\left(\eta_{k}\right)\right)\right. \\
& \left.-\Omega_{3}\left(A-\sum_{i=1}^{m} \omega_{i} \mathcal{I}_{0^{+}}^{\alpha-\beta_{i} ; \psi} h\left(\xi_{i}\right)\right)\right],
\end{aligned}
$$

where $\Omega$ is given by (10). Hence the solution $x$ follows by inserting $c_{1}$ and $c_{2}$ into (11). This implies that $x(\varsigma)$ satisfies (5).

On the contrary, it is easy to show by a straightforward procedure that $x(\varsigma)$, which is illustrated by (5), fulfills the given FBVP (3) in terms of supposed boundary conditions. Lemma 2.8 is proved.

\section{Existence results}

Set $F_{x}(\varsigma)=f\left(\varsigma, x(\theta \varsigma), \mathcal{I}_{0^{+}}^{q ; \psi} x(\varepsilon \varsigma)\right)$, where

$$
\mathcal{I}_{0^{+}}^{u ; \psi} F_{x}(c)=\frac{1}{\Gamma(u)} \int_{0}^{c} \psi^{\prime}(\tau)(\psi(c)-\psi(\tau))^{u-1} F_{x}(\tau) d \tau
$$

with $u=\left\{q, \beta_{j}\right\}$ and $c=\left\{\varsigma, \sigma, \theta_{j}\right\}$ for $j=1,2, \ldots, n$. According to Lemma 2.8, we define $\mathcal{Q}$ : $\mathbb{E} \rightarrow \mathbb{E}$ as

$$
\begin{aligned}
(\mathcal{Q} x)(\varsigma)= & \mathcal{I}_{0^{+}}^{\alpha ; \psi} F_{x}(\varsigma)+\frac{(\psi(\varsigma)-\psi(0))^{\gamma-1}}{\Omega \Gamma(\gamma)}\left[\Omega_{4}\left(A-\sum_{i=1}^{m} \omega_{i} \mathcal{I}_{0^{+}}^{\alpha-\beta_{i} ; \psi} F_{x}\left(\xi_{i}\right)\right)\right. \\
& \left.-\Omega_{2}\left(B-\sum_{j=1}^{n} \lambda_{j} \mathcal{I}_{0^{+}}^{\alpha-\mu_{j} ; \psi} F_{x}\left(\sigma_{j}\right)-\sum_{k=1}^{r} \delta_{k} \mathcal{I}_{0^{+}}^{\alpha ; \psi} F_{x}\left(\eta_{k}\right)\right)\right] \\
& +\frac{(\psi(\varsigma)-\psi(0))^{\gamma-2}}{\Omega \Gamma(\gamma-1)}\left[\Omega_{1}\left(B-\sum_{j=1}^{n} \lambda_{j} \mathcal{I}_{0^{+}}^{\alpha-\mu_{j} ; \psi} F_{x}\left(\sigma_{j}\right)-\sum_{k=1}^{r} \delta_{k} \mathcal{I}_{0^{+}}^{\alpha ; \psi} F_{x}\left(\eta_{k}\right)\right)\right. \\
& \left.-\Omega_{3}\left(A-\sum_{i=1}^{m} \omega_{i} \mathcal{I}_{0^{+}}^{\alpha-\beta_{i} ; \psi} F_{x}\left(\xi_{i}\right)\right)\right] .
\end{aligned}
$$

Note that the proposed $\psi$-Hilfer FBVP describing thermostat control model (3) involves solutions if and only if $\mathcal{Q}$ possesses fixed points. For brevity, we denote

$$
\begin{aligned}
& \Psi_{1}(X, u)=\frac{(\psi(X)-\psi(0))^{u}}{\Gamma(u+1)}, \\
& \Psi_{2}(U, V)=\frac{1}{|\Omega|}\left(|U| \Psi_{1}(T, \gamma-2)+|V| \Psi_{1}(T, \gamma-1)\right),
\end{aligned}
$$




$$
\begin{aligned}
\Lambda(U, V)= & U \Psi_{1}(T, \alpha)+V \Psi_{1}(T, q+\alpha) \\
& +\Psi_{2}\left(\Omega_{3}, \Omega_{4}\right) \sum_{i=1}^{m}\left|\omega_{i}\right|\left[U \Psi_{1}\left(\xi_{i}, \alpha-\beta_{i}\right)+V \Psi_{1}\left(\xi_{i}, q+\alpha-\beta_{i}\right)\right] \\
& +\Psi_{2}\left(\Omega_{1}, \Omega_{2}\right)\left(\sum_{j=1}^{n}\left|\lambda_{j}\right|\left[U \Psi_{1}\left(\sigma_{j}, \alpha-\mu_{j}\right)+V \Psi_{1}\left(\sigma_{j}, q+\alpha-\mu_{j}\right)\right]\right. \\
& \left.+\sum_{k=1}^{r}\left|\delta_{k}\right|\left[U \Psi_{1}\left(\eta_{k}, \alpha\right)+V \Psi_{1}\left(\eta_{k}, q+\alpha\right)\right]\right) .
\end{aligned}
$$

\subsection{Uniqueness property}

In the forthcoming first theorem, we will prove the uniqueness of solution for the $\psi$ Hilfer FBVP describing thermostat control model (3) by invoking the Banach principle (Lemma 3.1).

Lemma 3.1 ([36]) Let $S$ be a nonempty closed set contained in the Banach space $\mathbb{E}$. Then any contraction self-map $\mathcal{Q}$ on $\mathbb{E}$ has a unique fixed point.

Theorem 3.2 Letf $: \mathbb{J} \times \mathbb{R}^{2} \rightarrow \mathbb{R}$ be continuous and:

$\left(H_{1}\right)$ There exist $L_{1}, L_{2}>0$ such that

$$
\begin{aligned}
& \qquad\left|f\left(\varsigma, u_{1}, v_{1}\right)-f\left(\varsigma, u_{2}, v_{2}\right)\right| \leq L_{1}\left|u_{1}-u_{2}\right|+L_{2}\left|v_{1}-v_{2}\right| \\
& \text { for any } u_{l}, v_{l} \in \mathbb{R}, l=1,2 \text {, and } \varsigma \in \mathbb{J} \text {. } \\
& \text { If } \\
& \qquad \Lambda\left(L_{1}, L_{2}\right)<1,
\end{aligned}
$$

where $\Lambda(\cdot, \cdot)$ is defined in (15), then the $\psi$-Hilfer FBVP describing the thermostat control model (3) has a unique solution $x$ in $\mathbb{E}$.

Proof We convert the $\psi$-Hilfer FBVP describing the thermostat control model (3) into $x=\mathcal{Q} x$, where $\mathcal{Q}$ is given by (12). Obviously, the obtained fixed-points of $\mathcal{Q}$ are the possible solutions of the mentioned $\psi$-Hilfer FBVP (3). Following Lemma 3.1, we verify that $\mathcal{Q}$ admits a unique fixed point, which means that (3) involves exactly one solution.

Define a bounded, closed, convex, and nonempty subset $B_{r_{1}}:=\left\{x \in \mathbb{E}:\|x\| \leq r_{1}\right\}$ with

$$
r_{1} \geq \frac{\Lambda\left(M_{1}, 0\right)+|A| \Psi_{2}\left(\Omega_{3}, \Omega_{4}\right)+|B| \Psi_{2}\left(\Omega_{1}, \Omega_{2}\right)}{1-\Lambda\left(L_{1}, L_{2}\right)}, \quad \sup _{\varsigma \in \mathbb{J}} f(\varsigma, 0,0) \mid:=M_{1}<\infty
$$

where $\Omega_{i}$ for $i=1,2,3,4, \Psi_{2}(\cdot, \cdot)$, and $\Lambda(\cdot, \cdot)$ are given by (6)-(9), (14) and (15), respectively. We divide the proof into two steps.

Step I. $\mathcal{Q} B_{r_{1}} \subset B_{r_{1}}$.

Let $x \in B_{r_{1}}$ and $\varsigma \in \mathbb{J}$. Then

$$
\begin{aligned}
& |(\mathcal{Q} x)(\varsigma)| \\
& \quad \leq \mathcal{I}_{0^{+}}^{\alpha ; \psi}\left|F_{x}(s)\right|(T)+\frac{(\psi(T)-\psi(0))^{\gamma-1}}{|\Omega| \Gamma(\gamma)}
\end{aligned}
$$




$$
\begin{aligned}
& \times\left[\left|\Omega_{4}\right|\left(|A|+\sum_{i=1}^{m}\left|\omega_{i}\right| \mathcal{I}_{0^{+}}^{\alpha-\beta_{i} ; \psi}\left|F_{x}(s)\right|\left(\xi_{i}\right)\right)\right. \\
& \left.+\left|\Omega_{2}\right|\left(|B|+\sum_{j=1}^{n}\left|\lambda_{j}\right| \mathcal{I}_{0^{+}}^{\alpha-\mu_{j} ; \psi}\left|F_{x}(s)\right|\left(\sigma_{j}\right)+\sum_{k=1}^{r}\left|\delta_{k}\right| \mathcal{I}_{0^{+}}^{\alpha ; \psi}\left|F_{x}(s)\right|\left(\eta_{k}\right)\right)\right] \\
& +\frac{(\psi(T)-\psi(0))^{\gamma-2}}{|\Omega| \Gamma(\gamma-1)} \\
& \times\left[\left|\Omega_{1}\right|\left(|B|+\sum_{j=1}^{n}\left|\lambda_{j}\right| \mathcal{I}_{0^{+}}^{\alpha-\mu_{j} ; \psi}\left|F_{x}(s)\right|\left(\sigma_{j}\right)+\sum_{k=1}^{r}\left|\delta_{k}\right| \mathcal{I}_{0^{+}}^{\alpha ; \psi}\left|F_{x}(s)\right|\left(\eta_{k}\right)\right)\right. \\
& \left.+\left|\Omega_{3}\right|\left(|A|+\sum_{i=1}^{m}\left|\omega_{i}\right| \mathcal{I}_{0^{+}}^{\alpha-\beta_{i} ; \psi}\left|F_{x}(s)\right|\left(\xi_{i}\right)\right)\right] \\
& =\mathcal{I}_{0^{+}}^{\alpha ; \psi}\left|F_{x}(s)\right|(T)+\Psi_{2}\left(\Omega_{3}, \Omega_{4}\right)\left(|A|+\sum_{i=1}^{m}\left|\omega_{i}\right| \mathcal{I}_{0^{+}}^{\alpha-\beta_{i} ; \psi}\left|F_{x}(s)\right|\left(\xi_{i}\right)\right) \\
& +\Psi_{2}\left(\Omega_{1}, \Omega_{2}\right)\left(|B|+\sum_{j=1}^{n}\left|\lambda_{j}\right| \mathcal{I}_{0^{+}}^{\alpha-\mu_{j} ; \psi}\left|F_{x}(s)\right|\left(\sigma_{j}\right)+\sum_{k=1}^{r}\left|\delta_{k}\right| \mathcal{I}_{0^{+}}^{\alpha ; \psi}\left|F_{x}(s)\right|\left(\eta_{k}\right)\right) .
\end{aligned}
$$

By Proposition 2.5 we have

$$
\begin{aligned}
\mathcal{I}_{0^{+}}^{q ;}|x(\tau)|(s) & =\frac{1}{\Gamma(q)} \int_{0}^{s} \psi^{\prime}(\tau)(\psi(s)-\psi(\tau))^{q-1}|x(\tau)| d \tau \\
& \leq \frac{\|x\|}{\Gamma(q)} \int_{0}^{s} \psi^{\prime}(\tau)(\psi(s)-\psi(\tau))^{q-1} d \tau \\
& =\frac{(\psi(s)-\psi(0))^{q}}{\Gamma(q+1)}\|x\| .
\end{aligned}
$$

From $\left(H_{1}\right)$ we derive

$$
\begin{aligned}
\left|F_{x}(\varsigma)\right| & \leq\left|f\left(\varsigma, x(\theta \varsigma), \mathcal{I}_{0^{+}}^{q ; \psi} x(\varepsilon \varsigma)\right)-f(\varsigma, 0,0)\right|+|f(\varsigma, 0,0)| \\
& \leq L_{1}|x(\theta \varsigma)|+L_{2}\left|\mathcal{I}_{0^{+}}^{q ; \psi} x(\varepsilon \varsigma)\right|+M_{1} \\
& \leq L_{1}\|x\|+L_{2} \frac{(\psi(\varepsilon \varsigma)-\psi(0))^{q}}{\Gamma(q+1)}\|x\|+M_{1} \\
& \leq\left(L_{1}+L_{2} \frac{(\psi(\varsigma)-\psi(0))^{q}}{\Gamma(q+1)}\right)\|x\|+M_{1} .
\end{aligned}
$$

Then we compute that

$$
\begin{aligned}
& \mathcal{I}_{0^{+}}^{\alpha ; \psi}\left|F_{x}(s)\right|(T) \leq\left[L_{1} \Psi_{1}(T, \alpha)+L_{2} \Psi_{1}(T, q+\alpha)\right]\|x\|+M_{1} \Psi_{1}(T, \alpha), \\
& \mathcal{I}_{0^{+}}^{\alpha-\beta_{i} ; \psi}\left|F_{x}(s)\right|\left(\xi_{i}\right) \\
& \quad \leq\left[L_{1} \Psi_{1}\left(\xi_{i}, \alpha-\beta_{i}\right)+L_{2} \Psi_{1}\left(\xi_{i}, q+\alpha-\beta_{i}\right)\right]\|x\|+M_{1} \Psi_{1}\left(\xi_{i}, \alpha-\beta_{i}\right), \\
& \mathcal{I}_{0^{+}}^{\alpha-\mu_{j} ; \psi}\left|F_{x}(s)\right|\left(\sigma_{j}\right) \\
& \quad \leq\left[L_{1} \Psi_{1}\left(\sigma_{j}, \alpha-\mu_{j}\right)+L_{2} \Psi_{1}\left(\sigma_{j}, q+\alpha-\mu_{j}\right)\right]\|x\|+M_{1} \Psi_{1}\left(\sigma_{j}, \alpha-\mu_{j}\right),
\end{aligned}
$$




$$
\mathcal{I}_{0^{+}}^{\alpha ; \psi}\left|F_{x}(s)\right|\left(\eta_{k}\right) \leq\left[L_{1} \Psi_{1}\left(\eta_{k}, \alpha\right)+L_{2} \Psi_{1}\left(\eta_{k}, q+\alpha\right)\right]\|x\|+M_{1} \Psi_{1}\left(\eta_{k}, \alpha\right) .
$$

From (18), (19), (20), and (21) we obtain

$$
|(\mathcal{Q} x)(\varsigma)| \leq \Lambda\left(L_{1}, L_{2}\right) r_{1}+\Lambda\left(M_{1}, 0\right)+|A| \Psi_{2}\left(\Omega_{3}, \Omega_{4}\right)+|B| \Psi_{2}\left(\Omega_{1}, \Omega_{2}\right),
$$

which implies that $\|\mathcal{Q} x\| \leq r_{1}$. Hence $\mathcal{Q} B_{r_{1}} \subset B_{r_{1}}$.

Step II. The operator $\mathcal{Q}: \mathbb{E} \rightarrow \mathbb{E}$ is a contraction.

Let $x, y \in \mathbb{E}$. For each $\varsigma \in \mathbb{J}$, we get

$$
\begin{aligned}
|((\mathcal{Q} x)(\varsigma)-(\mathcal{Q} y)(s))| & \leq \mathcal{I}_{0^{+}}^{\alpha ; \psi}\left|F_{x}(s)-F_{y}(s)\right|(T)+\frac{(\psi(T)-\psi(0))^{\gamma-1}}{|\Omega| \Gamma(\gamma)}\left[\left|\Omega_{4}\right| \sum_{i=1}^{m}\left|\omega_{i}\right| \mathcal{I}_{0^{+}}^{\alpha-\beta_{i} ; \psi}\left|F_{x}(s)-F_{y}(s)\right|\left(\xi_{i}\right)\right. \\
& \left.+\left|\Omega_{2}\right|\left(\sum_{j=1}^{n}\left|\lambda_{j}\right| \mathcal{I}_{0^{+}}^{\alpha-\mu_{j} ; \psi}\left|F_{x}(s)-F_{y}(s)\right|\left(\sigma_{j}\right)+\sum_{k=1}^{r}\left|\delta_{k}\right| \mathcal{I}_{0^{+}}^{\alpha ; \psi}\left|F_{x}(s)-F_{y}(s)\right|\left(\eta_{k}\right)\right)\right] \\
& +\frac{(\psi(T)-\psi(0))^{\gamma-2}}{|\Omega| \Gamma(\gamma-1)}\left[| \Omega _ { 1 } | \left(\sum_{j=1}^{n}\left|\lambda_{j}\right| \mathcal{I}_{0^{+}}^{\alpha-\mu_{j} ; \psi}\left|F_{x}(s)-F_{y}(s)\right|\left(\sigma_{j}\right)\right.\right. \\
& \left.\left.+\sum_{k=1}^{r}\left|\delta_{k}\right| \mathcal{I}_{0^{+}}^{\alpha ; \psi}\left|F_{x}(s)-F_{y}(s)\right|\left(\eta_{k}\right)\right)+\left|\Omega_{3}\right| \sum_{i=1}^{m}\left|\omega_{i}\right| \mathcal{I}_{0^{+}}^{\alpha-\beta_{i} ; \psi}\left|F_{x}(s)-F_{y}(s)\right|\left(\xi_{i}\right)\right] \\
= & \mathcal{I}_{0^{+}}^{\alpha ; \psi}\left|F_{x}(s)-F_{y}(s)\right|(T)+\Psi_{2}\left(\Omega_{3}, \Omega_{4}\right) \sum_{i=1}^{m}\left|\omega_{i}\right| \mathcal{I}_{0^{+}}^{\alpha-\beta_{i} ; \psi}\left|F_{x}(s)-F_{y}(s)\right|\left(\xi_{i}\right) \\
& +\Psi_{2}\left(\Omega_{1}, \Omega_{2}\right)\left(\sum_{j=1}^{n}\left|\lambda_{j}\right| \mathcal{I}_{0^{+}}^{\alpha-\mu_{j} ; \psi}\left|F_{x}(s)-F_{y}(s)\right|\left(\sigma_{j}\right)\right. \\
& \left.+\sum_{k=1}^{r}\left|\delta_{k}\right| \mathcal{I}_{0^{+}}^{\alpha ; \psi}\left|F_{x}(s)-F_{y}(s)\right|\left(\eta_{k}\right)\right) .
\end{aligned}
$$

From $\left(H_{1}\right)$ we obtain

$$
\begin{aligned}
\left|F_{x}(\varsigma)-F_{y}(\varsigma)\right| & =\left|f\left(\varsigma, x(\theta \varsigma), \mathcal{I}_{0^{+}}^{q ; \psi} x(\varepsilon \varsigma)\right)-f\left(\varsigma, y(\theta \varsigma), \mathcal{I}_{0^{+}}^{q ; \psi} y(\varepsilon \varsigma)\right)\right| \\
& \leq L_{1}|x(\theta \varsigma)-y(\theta \varsigma)|+L_{2}\left|\mathcal{I}_{0^{+}}^{q ; \psi} x(\varepsilon \varsigma)-\mathcal{I}_{0^{+}}^{q ; \psi} x(\varepsilon \varsigma)\right| \\
& \leq L_{1}\|x-y\|+L_{2} \frac{(\psi(\varepsilon \varsigma)-\psi(0))^{q}}{\Gamma(q+1)}\|x-y\| \\
& \leq\left(L_{1}+L_{2} \frac{(\psi(\varsigma)-\psi(0))^{q}}{\Gamma(q+1)}\right)\|x-y\| .
\end{aligned}
$$

Then by substituting (23) into (22) we get

$$
\begin{aligned}
& |(\mathcal{Q} x)(\varsigma)-(\mathcal{Q} y)(\varsigma)| \\
& \quad \leq\left[L_{1} \Psi_{1}(T, \alpha)+L_{2} \Psi_{1}(T, q+\alpha)\right]\|x-y\| \\
& \quad+\Psi_{2}\left(\Omega_{1}, \Omega_{2}\right)\left(\sum_{j=1}^{n}\left|\lambda_{j}\right|\left[L_{1} \Psi_{1}\left(\sigma_{j}, \alpha-\mu_{j}\right)+L_{2} \Psi_{1}\left(\sigma_{j}, q+\alpha-\mu_{j}\right)\right]\|x-y\|\right.
\end{aligned}
$$




$$
\begin{aligned}
& \left.+\sum_{k=1}^{r}\left|\delta_{k}\right|\left[L_{1} \Psi_{1}\left(\eta_{k}, \alpha\right)+L_{2} \Psi_{1}\left(\eta_{k}, q+\alpha\right)\right]\|x-y\|\right)+\Psi_{2}\left(\Omega_{3}, \Omega_{4}\right) \\
& \times\left(\sum_{i=1}^{m}\left|\omega_{i}\right|\left[L_{1} \Psi_{1}\left(\xi_{i}, \alpha-\beta_{i}\right)+L_{2} \Psi_{1}\left(\xi_{i}, q+\alpha-\beta_{i}\right)\right]\|x-y\|\right),
\end{aligned}
$$

which illustrates that $\|\mathcal{Q} x-\mathcal{Q} y\| \leq \Lambda\left(L_{1}, L_{2}\right)\|x-y\|$. In view of the condition $\Lambda\left(L_{1}, L_{2}\right)<1$, we get that $\mathcal{Q}$ is a contraction. Hence by Lemma 3.1 we get that the solution $x \in \mathbb{E}$ is unique on $\mathbb{J}$ for the supposed $\psi$-Hilfer FBVP describing the thermostat control model (3). The proof of the theorem is completed.

\subsection{Existence property}

The second result is obtained by invoking the Schaefer fixed-point theorem (Lemma 3.3).

Lemma 3.3 ([36]) Let $\mathcal{Q}: \mathbb{E} \rightarrow \mathbb{E}$ be a completely continuous self-map on the Banach space $\mathbb{E}$, and let $\mathbb{P}=\{x \in \mathbb{E}: x=\kappa \mathcal{Q} x, 0<\kappa \leq 1\}$ be bounded. Then a fixed-point exists for $\mathcal{Q}$ in $\mathbb{E}$.

Theorem 3.4 Let $f: \mathbb{J} \times \mathbb{R}^{2} \rightarrow \mathbb{R}$ be continuous. Suppose that:

$\left(H_{2}\right)$ There exist nonnegative continuous functions $k_{1}, k_{2}, k_{3} \in \mathcal{C}\left(\mathbb{J}, \mathbb{R}^{+} \cup\{0\}\right)$ such that

$$
|f(\varsigma, u, v)| \leq k_{1}(\varsigma)+k_{2}(\varsigma)|u|+k_{3}(\varsigma)|v|, \quad u, v \in \mathbb{R}, \varsigma \in \mathbb{J}
$$

with $k_{1}^{*}=\sup _{\varsigma \in \mathbb{J}}\left\{k_{1}(\varsigma)\right\}, k_{2}^{*}=\sup _{\varsigma \in \mathbb{J}}\left\{k_{2}(\varsigma)\right\}$, and $k_{3}^{*}=\sup _{\varsigma \in \mathbb{J}}\left\{k_{3}(\varsigma)\right\}$.

Then a solution exists on $\mathbb{I}$ for the supposed $\psi$-Hilfer FBVP describing the thermostat control model (3).

Proof We divide the proof into four steps.

Step I. $\mathcal{Q}$ is continuous.

Let a sequence $\left\{x_{n}\right\} \subset \mathbb{E}$ be such that $x_{n} \rightarrow x$ in $\mathbb{E}$. Then, for every $\varsigma \in \mathbb{J}$, we obtain

$$
\begin{aligned}
\left|\left(\mathcal{Q} x_{n}\right)(\varsigma)-(\mathcal{Q} x)(\varsigma)\right| & \\
\leq & \mathcal{I}_{0^{+}}^{\alpha ; \psi}\left|F_{x_{n}}(s)-F_{x}(s)\right|(T)+\frac{(\psi(T)-\psi(0))^{\gamma-1}}{|\Omega| \Gamma(\gamma)} \\
& \times\left[\left|\Omega_{4}\right| \sum_{i=1}^{m}\left|\omega_{i}\right| \mathcal{I}_{0^{+}}^{\alpha-\beta_{i} ; \psi}\left|F_{x_{n}}(s)-F_{x}(s)\right|\left(\xi_{i}\right)\right. \\
+ & \left|\Omega_{2}\right|\left(\sum_{j=1}^{n}\left|\lambda_{j}\right| \mathcal{I}_{0^{+}}^{\alpha-\mu_{j} ; \psi}\left|F_{x_{n}}(s)-F_{x}(s)\right|\left(\sigma_{j}\right)\right. \\
+ & \left.\left.\sum_{k=1}^{r}\left|\delta_{k}\right| \mathcal{I}_{0^{+}}^{\alpha ; \psi}\left|F_{x_{n}}(s)-F_{x}(s)\right|\left(\eta_{k}\right)\right)\right] \\
+ & \frac{(\psi(\varsigma)-\psi(0))^{\gamma-2}}{|\Omega| \Gamma(\gamma-1)}\left[| \Omega _ { 1 } | \left(\sum_{j=1}^{n}\left|\lambda_{j}\right| \mathcal{I}_{0^{+}}^{\alpha-\mu_{j} ; \psi}\left|F_{x_{n}}(s)-F_{x}(s)\right|\left(\sigma_{j}\right)\right.\right. \\
+ & \left.\sum_{k=1}^{r}\left|\delta_{k}\right| \mathcal{I}_{0^{+}}^{\alpha ; \psi}\left|F_{x_{n}}(s)-F_{x}(s)\right|\left(\eta_{k}\right)\right)
\end{aligned}
$$




$$
\begin{aligned}
& \left.+\left|\Omega_{3}\right| \sum_{i=1}^{m}\left|\omega_{i}\right| \mathcal{I}_{0^{+}}^{\alpha-\beta_{i} ; \psi}\left|F_{x_{n}}(s)-F_{x}(s)\right|\left(\xi_{i}\right)\right] \\
= & \mathcal{I}_{0^{+}}^{\alpha ; \psi}\left|F_{x_{n}}(s)-F_{x}(s)\right|(T)+\Psi_{2}\left(\Omega_{1}, \Omega_{2}\right)\left(\sum_{j=1}^{n}\left|\lambda_{j}\right| \mathcal{I}_{0^{+}}^{\alpha-\mu_{j} ; \psi}\left|F_{x_{n}}(s)-F_{x}(s)\right|\left(\sigma_{j}\right)\right. \\
& \left.+\sum_{k=1}^{r}\left|\delta_{k}\right| \mathcal{I}_{0^{+}}^{\alpha ; \psi}\left|F_{x_{n}}(s)-F_{x}(s)\right|\left(\eta_{k}\right)\right) \\
& +\Psi_{2}\left(\Omega_{3}, \Omega_{4}\right) \sum_{i=1}^{m}\left|\omega_{i}\right| \mathcal{I}_{0^{+}}^{\alpha-\beta_{i} ; \psi}\left|F_{x_{n}}(s)-F_{x}(s)\right|\left(\xi_{i}\right) \\
\leq & {\left[\Psi_{1}(T, \alpha)+\Psi_{2}\left(\Omega_{1}, \Omega_{2}\right)\left(\sum_{j=1}^{n}\left|\lambda_{j}\right| \Psi_{1}\left(\sigma_{j}, \alpha-\mu_{j}\right)+\sum_{k=1}^{r}\left|\delta_{k}\right| \Psi_{1}\left(\eta_{k}, \alpha\right)\right)\right.} \\
& \left.+\Psi_{2}\left(\Omega_{3}, \Omega_{4}\right) \sum_{i=1}^{m}\left|\omega_{i}\right| \Psi_{1}\left(\xi_{i}, \alpha-\beta_{i}\right)\right]\left\|F_{x_{n}}-F_{x}\right\| .
\end{aligned}
$$

Since the continuity of $f$ implies the continuity of $F_{x}$, we obtain

$$
\left\|F_{x_{n}}-F_{x}\right\| \rightarrow 0 \text { as } n \rightarrow \infty
$$

and therefore $\mathcal{Q}$ is continuous.

Step II. $\mathcal{Q}$ maps bounded sets to bounded ones contained in $\mathbb{E}$.

For $r_{2}>0$, there is $N>0$ such that, for every $x \in B_{r_{2}}=\left\{x \in \mathbb{E}:\|x\| \leq r_{2}\right\}$, we have $\|\mathcal{Q} x\| \leq$ $N$.

Indeed, for any $\varsigma \in \mathbb{J}$ and $x \in B_{r_{2}}$, we have

$$
\begin{aligned}
|(\mathcal{Q} x)(\varsigma)| & \leq \mathcal{I}_{0^{+}}^{\alpha ; \psi}\left|F_{x}(s)\right|(T) \\
& +\frac{(\psi(T)-\psi(0))^{\gamma-1}}{|\Omega| \Gamma(\gamma)}\left[\left|\Omega_{4}\right|\left(|A|+\sum_{i=1}^{m}\left|\omega_{i}\right| \mathcal{I}_{0^{+}}^{\alpha-\beta_{i} ; \psi}\left|F_{x}(s)\right|\left(\xi_{i}\right)\right)\right. \\
& \left.+\left|\Omega_{2}\right|\left(|B|+\sum_{j=1}^{n}\left|\lambda_{j}\right| \mathcal{I}_{0^{+}}^{\alpha-\mu_{j} ; \psi}\left|F_{x}(s)\right|\left(\sigma_{j}\right)+\sum_{k=1}^{r}\left|\delta_{k}\right| \mathcal{I}_{0^{+}}^{\alpha ; \psi}\left|F_{x}(s)\right|\left(\eta_{k}\right)\right)\right] \\
& +\frac{(\psi(T)-\psi(0))^{\gamma-2}}{|\Omega| \Gamma(\gamma-1)} \\
& \times\left[\left|\Omega_{1}\right|\left(|B|+\sum_{j=1}^{n}\left|\lambda_{j}\right| \mathcal{I}_{0^{+}}^{\alpha-\mu_{j} ; \psi}\left|F_{x}(s)\right|\left(\sigma_{j}\right)+\sum_{k=1}^{r}\left|\delta_{k}\right| \mathcal{I}_{0^{+}}^{\alpha ; \psi}\left|F_{x}(s)\right|\left(\eta_{k}\right)\right)\right. \\
& \left.+\left|\Omega_{3}\right|\left(|A|+\sum_{i=1}^{m}\left|\omega_{i}\right| \mathcal{I}_{0^{+}}^{\alpha-\beta_{i} ; \psi}\left|F_{x}(s)\right|\left(\xi_{i}\right)\right)\right] \\
= & \mathcal{I}_{0^{+}}^{\alpha ; \psi}\left|F_{x}(s)\right|(T)+\Psi_{2}\left(\Omega_{3}, \Omega_{4}\right)\left(|A|+\sum_{i=1}^{m}\left|\omega_{i}\right| \mathcal{I}_{0^{+}}^{\alpha-\beta_{i} ; \psi}\left|F_{x}(s)\right|\left(\xi_{i}\right)\right) \\
& +\Psi_{2}\left(\Omega_{1}, \Omega_{2}\right)
\end{aligned}
$$




$$
\times\left(|B|+\sum_{j=1}^{n}\left|\lambda_{j}\right| \mathcal{I}_{0^{+}}^{\alpha-\mu_{j} ; \psi}\left|F_{x}(s)\right|\left(\sigma_{j}\right)+\sum_{k=1}^{r}\left|\delta_{k}\right| \mathcal{I}_{0^{+}}^{\alpha ; \psi}\left|F_{x}(s)\right|\left(\eta_{k}\right)\right) .
$$

It follows from $\left(H_{2}\right)$ that

$$
\begin{aligned}
\left|F_{x}(\varsigma)\right| & \left.\leq k_{1}(\varsigma)+k_{2}(\varsigma)|x(\theta \varsigma)|+k_{3}(\varsigma) \mid \mathcal{I}_{0^{+}}^{q ; \psi} x(\varepsilon \varsigma)\right) \mid \\
& \leq k_{1}^{*}+\left(k_{2}^{*}+k_{3}^{*} \frac{(\psi(\varsigma)-\psi(0))^{q}}{\Gamma(q+1)}\right)\|x\| .
\end{aligned}
$$

Substituting (25) into (24), we get

$$
\begin{aligned}
|(\mathcal{Q} x)(\varsigma)| & \\
\leq & {\left[k_{2}^{*} \Psi_{1}(T, \alpha)+k_{3}^{*} \Psi_{1}(T, q+\alpha)\right]\|x\| } \\
& +\Psi_{2}\left(\Omega_{3}, \Omega_{4}\right) \sum_{i=1}^{m}\left|\omega_{i}\right|\left[k_{2}^{*} \Psi_{1}\left(\xi_{i}, \alpha-\beta_{i}\right)+k_{3}^{*} \Psi_{1}\left(\xi_{i}, q+\alpha-\beta_{i}\right)\right]\|x\| \\
& +\Psi_{2}\left(\Omega_{1}, \Omega_{2}\right)\left(\sum_{j=1}^{n}\left|\lambda_{j}\right|\left[k_{2}^{*} \Psi_{1}\left(\sigma_{j}, \alpha-\mu_{j}\right)+k_{3}^{*} \Psi_{1}\left(\sigma_{j}, q+\alpha-\mu_{j}\right)\right]\|x\|\right. \\
& \left.+\sum_{k=1}^{r}\left|\delta_{k}\right|\left[k_{2}^{*} \Psi_{1}\left(\eta_{k}, \alpha\right)+k_{3}^{*} \Psi_{1}\left(\eta_{k}, q+\alpha\right)\right]\|x\|\right) \\
& +|A| \Psi_{2}\left(\Omega_{3}, \Omega_{4}\right)+|B| \Psi_{2}\left(\Omega_{1}, \Omega_{2}\right) \\
& +k_{1}^{*} \Psi_{1}(T, \alpha)+k_{1}^{*} \Psi_{2}\left(\Omega_{3}, \Omega_{4}\right) \sum_{i=1}^{m}\left|\omega_{i}\right| \Psi_{1}\left(\xi_{i}, \alpha-\beta_{i}\right) \\
& +k_{1}^{*} \Psi_{2}\left(\Omega_{1}, \Omega_{2}\right)\left(\sum_{j=1}^{n}\left|\lambda_{j}\right| \Psi_{1}\left(\sigma_{j}, \alpha-\mu_{j}\right)+\sum_{k=1}^{r}\left|\delta_{k}\right| \Psi_{1}\left(\eta_{k}, \alpha\right)\right)
\end{aligned}
$$

from which we get

$$
\|\mathcal{Q} x\| \leq \Lambda\left(k_{2}^{*}, k_{3}^{*}\right) r_{2}+\Lambda\left(k_{1}^{*}, 0\right)+|A| \Psi_{2}\left(\Omega_{3}, \Omega_{4}\right)+|B| \Psi_{2}\left(\Omega_{1}, \Omega_{2}\right):=N .
$$

Step III. $\mathcal{Q}$ maps bounded sets to equicontinuous ones contained in $\mathbb{E}$.

For $0 \leq \varsigma_{1}<\varsigma_{2} \leq T$ and $x \in B_{r_{2}}$, since $f$ is bounded on the compact set $\mathbb{J} \times B_{r_{2}}$, we have

$$
\begin{aligned}
& \left|(\mathcal{Q} x)\left(\varsigma_{2}\right)-(\mathcal{Q} x)\left(\varsigma_{1}\right)\right| \\
& \leq \frac{\left|\left(\psi\left(\varsigma_{2}\right)-\psi(0)\right)^{\gamma-1}-\left(\psi\left(\varsigma_{1}\right)-\psi(0)\right)^{\gamma-1}\right|}{|\Omega| \Gamma(\gamma)} \\
& \quad \times\left[\left|\Omega_{4}\right|\left(|A|+\sum_{i=1}^{m}\left|\omega_{i}\right| \mathcal{I}_{0^{+}}^{\alpha-\beta_{i} ; \psi}\left|F_{x}(s)\right|\left(\xi_{i}\right)\right)\right. \\
& \left.\quad+\left|\Omega_{2}\right|\left(|B|+\sum_{j=1}^{n}\left|\lambda_{j}\right| \mathcal{I}_{0^{+}}^{\alpha-\mu_{j} ; \psi}\left|F_{x}(s)\right|\left(\sigma_{j}\right)+\sum_{k=1}^{r}\left|\delta_{k}\right| \mathcal{I}_{0^{+}}^{\alpha ; \psi}\left|F_{x}(s)\right|\left(\eta_{k}\right)\right)\right] \\
& \quad+\frac{\left|\left(\psi\left(\varsigma_{2}\right)-\psi(0)\right)^{\gamma-2}-\left(\psi\left(\varsigma_{1}\right)-\psi(0)\right)^{\gamma-2}\right|}{|\Omega| \Gamma(\gamma-1)}
\end{aligned}
$$




$$
\begin{aligned}
& \times\left[| \Omega _ { 1 } | \left(|B|+\sum_{j=1}^{n}\left|\lambda_{j}\right| \mathcal{I}_{0^{+}}^{\alpha-\mu_{j} ; \psi}\left|F_{x}(s)\right|\left(\sigma_{j}\right)\right.\right. \\
& \left.\left.+\sum_{k=1}^{r}\left|\delta_{k}\right| \mathcal{I}_{0^{+}}^{\alpha ; \psi}\left|F_{x}(s)\right|\left(\eta_{k}\right)\right)+\left|\Omega_{3}\right|\left(|A|+\sum_{i=1}^{m}\left|\omega_{i}\right| \mathcal{I}_{0^{+}}^{\alpha-\beta_{i} ; \psi}\left|F_{x}(s)\right|\left(\xi_{i}\right)\right)\right] \\
& +\left|\mathcal{I}_{0^{+}}^{\alpha ; \psi} F_{x}(s)\left(\varsigma_{2}\right)-\mathcal{I}_{0^{+}}^{\alpha ; \psi} F_{x}(s)\left(\varsigma_{1}\right)\right| .
\end{aligned}
$$

By setting $\sup _{(\varsigma, u, v) \in \mathbb{J} \times B_{r_{2}}^{2}}|f(\varsigma, u, v)|=\widehat{f}<\infty$ it follows that

$$
\begin{aligned}
\mid(\mathcal{Q} x) & \left(\varsigma_{2}\right)-(\mathcal{Q} x)\left(\varsigma_{1}\right) \mid \\
\leq & \frac{\widehat{f}}{\Gamma(\alpha+1)} \\
& \times\left[\left(\psi\left(\varsigma_{2}\right)-\psi\left(\varsigma_{1}\right)\right)^{\alpha}+\left|\left(\psi\left(\varsigma_{2}\right)-\psi(0)\right)^{\alpha}-\left(\psi\left(\varsigma_{2}\right)-\psi\left(\varsigma_{1}\right)\right)^{\alpha}-\left(\psi\left(\varsigma_{1}\right)-\psi(0)\right)^{\alpha}\right|\right] \\
& +\frac{\left|\left(\psi\left(\varsigma_{2}\right)-\psi(0)\right)^{\gamma-1}-\left(\psi\left(\varsigma_{1}\right)-\psi(0)\right)^{\gamma-1}\right|}{|\Omega| \Gamma(\gamma)} \\
& \times\left[\left|\Omega_{4}\right|\left(|A|+\widehat{f} \sum_{i=1}^{m} \frac{\left|\omega_{i}\right|\left(\psi\left(\xi_{i}\right)-\psi(0)\right)^{\alpha-\beta_{i}}}{\Gamma\left(\alpha-\beta_{i}+1\right)}\right)\right. \\
& \left.+\left|\Omega_{2}\right|\left(|B|+\widehat{f} \sum_{j=1}^{n} \frac{\left|\lambda_{j}\right|\left(\psi\left(\sigma_{j}\right)-\psi(0)\right)^{\alpha-\mu_{j}}}{\Gamma\left(\alpha-\mu_{j}+1\right)}+\widehat{f} \sum_{k=1}^{r} \frac{\left|\delta_{k}\right|\left(\psi\left(\eta_{k}\right)-\psi(0)\right)^{\alpha}}{\Gamma(\alpha+1)}\right)\right] \\
+ & \frac{\left(\psi\left(\varsigma_{2}\right)-\psi(0)\right)^{\gamma-2}-\left(\psi\left(\varsigma_{1}\right)-\psi(0)\right)^{\gamma-2}}{|\Omega| \Gamma(\gamma-1)} \\
& \times\left[| \Omega _ { 1 } | \left(|B|+\widehat{f} \sum_{j=1}^{n} \frac{\left|\lambda_{j}\right|\left(\psi\left(\sigma_{j}\right)-\psi(0)\right)^{\alpha-\mu_{j}}}{\Gamma\left(\alpha-\mu_{j}+1\right)}\right.\right. \\
& \left.+\widehat{f} \sum_{k=1}^{r} \frac{\left|\delta_{k}\right|\left(\psi\left(\eta_{k}\right)-\psi(0)\right)^{\alpha}}{\Gamma(\alpha+1)}\right) \\
+ & \left|\Omega_{3}\right|\left(|A|+\widehat{f} \sum_{i=1}^{m} \frac{\left|\omega_{i}\right|\left(\psi\left(\xi_{i}\right)-\psi(0)\right)^{\alpha-\beta_{i}}}{\Gamma\left(\alpha-\beta_{i}+1\right)}\right)
\end{aligned}
$$

Clearly, the right-hand side of (26) is independent of the unknown variable $x$ and approaches 0 as $\varsigma_{2} \rightarrow \varsigma_{1}$. Then the operator $\mathcal{Q}$ is equicontinuous. So, the operator $\mathcal{Q}$ admits the relative compactness on $B_{r_{2}}$, and the Arzelá-Ascoli theorem gives the complete continuity of $\mathcal{Q}$.

Step IV. $\mathbb{P}=\{x \in \mathbb{E}: x=\tau \mathcal{Q} x, \tau \in(0,1]\}$ is bounded.

Let $x \in \mathbb{P}$. Then $x=\tau \mathcal{Q} x$ for some $\tau \in(0,1]$. From $\left(H_{2}\right)$, for each $\varsigma \in \mathbb{J}$, we get the estimate

$$
\begin{aligned}
x(\varsigma)= & \varsigma\left(\mathcal{I}_{0^{+}}^{\alpha ; \psi} F_{x}(\varsigma)+\frac{(\psi(\varsigma)-\psi(0))^{\gamma-1}}{\Omega \Gamma(\gamma)}\left[\Omega_{4}\left(A-\sum_{i=1}^{m} \omega_{i} \mathcal{I}_{0^{+}}^{\alpha-\beta_{i} ; \psi} F_{x}\left(\xi_{i}\right)\right)\right.\right. \\
& \left.-\Omega_{2}\left(B-\sum_{j=1}^{n} \lambda_{j} \mathcal{I}_{0^{+}}^{\alpha-\mu_{j} ; \psi} F_{x}\left(\sigma_{j}\right)-\sum_{k=1}^{r} \delta_{k} \mathcal{I}_{0^{+}}^{\alpha ; \psi} F_{x}\left(\eta_{k}\right)\right)\right]
\end{aligned}
$$




$$
\begin{aligned}
& +\frac{(\psi(\varsigma)-\psi(0))^{\gamma-2}}{\Omega \Gamma(\gamma-1)}\left[\Omega_{1}\left(B-\sum_{j=1}^{n} \lambda_{j} \mathcal{I}_{0^{+}}^{\alpha-\mu_{j} ; \psi} F_{x}\left(\sigma_{j}\right)-\sum_{k=1}^{r} \delta_{k} \mathcal{I}_{0^{+}}^{\alpha ; \psi} F_{x}\left(\eta_{k}\right)\right)\right. \\
& \left.\left.-\Omega_{3}\left(A-\sum_{i=1}^{m} \omega_{i} \mathcal{I}_{0^{+}}^{\alpha-\beta_{i} ; \psi} F_{x}\left(\xi_{i}\right)\right)\right]\right) .
\end{aligned}
$$

From Step II, for any $\varsigma \in \mathbb{J}$, we get that $\|\mathcal{Q} x\| \leq N<\infty$. Hence the set $\mathbb{P}$ is bounded.

Using Theorem 3.4, we see that we can find $N>0$ such that $\|x\| \leq N<\infty$. By Lemma 3.3 we find at least one fixed-point for $\mathcal{Q}$, which is the corresponding solution of the suggested $\psi$-Hilfer FBVP describing the thermostat control model (3).

Finally, by applying the Krasnoselskii fixed-point criterion we will prove the existence of a solution.

Lemma 3.5 ([37]) Let $\mathcal{M}$ be a closed convex bounded nonempty set in a Banach space. Let $\mathcal{Q}_{1}, \mathcal{Q}_{2}$ be such that (i) $\mathcal{Q}_{1} x+\mathcal{Q}_{2} y \in \mathcal{M}$ for $x, y \in \mathcal{M}$; (ii) $\mathcal{Q}_{1}$ is compact and continuous; and (iii) $\mathcal{Q}_{2}$ is a contraction. Then there exists $z \in \mathcal{M}$ such that $z=\mathcal{Q}_{1} z+\mathcal{Q}_{2} z$.

Theorem 3.6 Letf $: \mathbb{I} \times \mathbb{R}^{2} \rightarrow \mathbb{R}$ be a continuous functions satisfying $\left(H_{1}\right)$. Moreover, suppose that:

$\left(H_{3}\right) f(\varsigma, u, v) \leq \Theta(\varsigma),(\varsigma, u, v) \in \mathbb{J} \times \mathbb{R}^{2}$, and $\Theta(\varsigma) \in \mathcal{C}\left(\mathbb{J}, \mathbb{R}^{+}\right)$.

If

$$
\Lambda\left(L_{1}, L_{2}\right)-L_{1} \Psi_{1}(T, \alpha)-L_{2} \Psi_{1}(T, q+\alpha)<1,
$$

where $\Lambda_{1}(\cdot, \cdot)$ and $\Psi_{1}(\cdot, \cdot)$ are given by (15) and (13), respectively, then the $\psi$-Hilfer FBVP describing the thermostat control model (3) admits a solution on $\mathbb{J}$.

Proof Let $\sup _{\varsigma \in \mathbb{J}}|\Theta(\varsigma)|=\Theta^{*}$ and set $B_{r_{3}}:=\left\{x \in \mathbb{E}:\|x\| \leq r_{3}\right\}$, where

$$
r_{3} \geq|A| \Psi_{2}\left(\Omega_{3}, \Omega_{4}\right)+|B| \Psi_{2}\left(\Omega_{1}, \Omega_{2}\right)+\Lambda_{1}\left(\Theta^{*}, 0\right) .
$$

We define $\mathcal{Q}_{1}$ and $\mathcal{Q}_{2}$ on $B_{r_{3}}$ by

$$
\begin{aligned}
\left(\mathcal{Q}_{1} x\right)(\varsigma)= & \mathcal{I}_{0^{+}}^{\alpha ; \psi} F_{x}(\varsigma), \quad \varsigma \in \mathbb{J}, \\
\left(\mathcal{Q}_{2} x\right)(\varsigma)= & \frac{(\psi(\varsigma)-\psi(0))^{\gamma-1}}{\Omega \Gamma(\gamma)}\left[\Omega_{4}\left(A-\sum_{i=1}^{m} \omega_{i} \mathcal{I}_{0^{+}}^{\alpha-\beta_{i} ; \psi} F_{x}\left(\xi_{i}\right)\right)\right. \\
& \left.-\Omega_{2}\left(B-\sum_{j=1}^{n} \lambda_{j} \mathcal{I}_{0^{+}}^{\alpha-\mu_{j} ; \psi} F_{x}\left(\sigma_{j}\right)-\sum_{k=1}^{r} \delta_{k} \mathcal{I}_{0^{+}}^{\alpha ; \psi} F_{x}\left(\eta_{k}\right)\right)\right] \\
& +\frac{(\psi(\varsigma)-\psi(0))^{\gamma-2}}{\Omega \Gamma(\gamma-1)}\left[\Omega_{1}\left(B-\sum_{j=1}^{n} \lambda_{j} \mathcal{I}_{0^{+}}^{\alpha-\mu_{j} ; \psi} F_{x}\left(\sigma_{j}\right)-\sum_{k=1}^{r} \delta_{k} \mathcal{I}_{0^{+}}^{\alpha ; \psi} F_{x}\left(\eta_{k}\right)\right)\right. \\
& \left.-\Omega_{3}\left(A-\sum_{i=1}^{m} \omega_{i} \mathcal{I}_{0^{+}}^{\alpha-\beta_{i} ; \psi} F_{x}\left(\xi_{i}\right)\right)\right], \quad(\varsigma \in \mathbb{J}) .
\end{aligned}
$$

Note that $\mathcal{Q}=\mathcal{Q}_{1}+\mathcal{Q}_{2}$. 
For any $x, y \in B_{r_{3}}$, we have

$$
\begin{aligned}
& \left|\left(\mathcal{Q}_{1} x\right)(\varsigma)+\left(\mathcal{Q}_{2} y\right)(\varsigma)\right| \\
& \leq \mathcal{I}_{0^{+}}^{\alpha ; \psi}\left|F_{x}(s)\right|(T)+\Psi_{2}\left(\Omega_{3}, \Omega_{4}\right)\left(|A|+\sum_{i=1}^{m}\left|\omega_{i}\right| \mathcal{I}_{0^{+}}^{\alpha-\beta_{i} ; \psi}\left|F_{y}(s)\right|\left(\xi_{i}\right)\right) \\
& \quad+\Psi_{2}\left(\Omega_{1}, \Omega_{2}\right)\left(|B|+\sum_{j=1}^{n}\left|\lambda_{j}\right| \mathcal{I}_{0^{+}}^{\alpha-\mu_{j} ; \psi}\left|F_{y}(s)\right|\left(\sigma_{j}\right)+\sum_{k=1}^{r}\left|\delta_{k}\right| \mathcal{I}_{0^{+}}^{\alpha ; \psi}\left|F_{y}(s)\right|\left(\eta_{k}\right)\right) \\
& \leq|A| \Psi_{2}\left(\Omega_{3}, \Omega_{4}\right)+|B| \Psi_{2}\left(\Omega_{1}, \Omega_{2}\right) \\
& \quad+\Theta^{*} \Psi_{1}(T, \alpha)+\Theta^{*} \Psi_{2}\left(\Omega_{3}, \Omega_{4}\right) \sum_{i=1}^{m}\left|\omega_{i}\right| \Psi_{1}\left(\xi_{i}, \alpha-\beta_{i}\right) \\
& \quad+\Theta^{*} \Psi_{2}\left(\Omega_{1}, \Omega_{2}\right)\left(\sum_{j=1}^{n}\left|\lambda_{j}\right| \Psi_{1}\left(\sigma_{j}, \alpha-\mu_{j}\right)+\sum_{k=1}^{r}\left|\delta_{k}\right| \Psi_{1}\left(\eta_{k}, \alpha\right)\right) \\
& =|A| \Psi_{2}\left(\Omega_{3}, \Omega_{4}\right)+|B| \Psi_{2}\left(\Omega_{1}, \Omega_{2}\right)+\Lambda\left(\Theta^{*}, 0\right) \leq r_{3} .
\end{aligned}
$$

This implies that $\mathcal{Q}_{1} x+\mathcal{Q}_{2} x \in B_{r_{3}}$, which satisfies Lemma 3.5(i).

Next, we show that Lemma 3.5(ii) is fulfilled.

Let $\left\{x_{n}\right\} \subset \mathbb{E}$ be such that $x_{n} \rightarrow x$ in $\mathbb{E}$. For each $\varsigma \in \mathbb{J}$, we have

$$
\left|\left(\mathcal{Q}_{1} x_{n}\right)(\varsigma)-\left(\mathcal{Q}_{1} x\right)(\varsigma)\right| \leq \mathcal{I}_{0^{+}}^{\alpha ; \psi}\left|F_{x_{n}}(s)-F_{x}(s)\right|(T) \leq \frac{(\psi(T)-\psi(0))^{\alpha}}{\Gamma(\alpha+1)}\left\|F_{x_{n}}-F_{x}\right\|
$$

Due to the continuity of $f$, this implies the continuity of $F_{x}$. Hence we obtain

$$
\left\|F_{x_{n}}-F_{x}\right\| \rightarrow 0 \quad \text { as } n \rightarrow \infty
$$

Thus $Q_{1}$ is continuous. Also, the set $Q_{1} B_{r_{3}}$ is uniformly bounded since

$$
\left\|\mathcal{Q}_{1} x\right\| \leq \Theta^{*} \Psi_{1}(T, \alpha)
$$

Now we prove the compactness of $\mathcal{Q}_{1}$. Setting $\sup _{(\varsigma, u, v) \in \mathbb{J} \times B_{r_{3}}^{2}}|f(\varsigma, u, v)|=f^{*}<\infty$, for all $\varsigma_{1}, \varsigma_{2} \in \mathbb{J}$ with $0 \leq \varsigma_{1}<\varsigma_{2} \leq T$, we have (see Step III of Theorem 3.4)

$$
\begin{aligned}
&\left|\left(\mathcal{Q}_{1} x\right)\left(\varsigma_{2}\right)-\left(\mathcal{Q}_{1} x\right)\left(\varsigma_{1}\right)\right| \\
& \leq \mid\left|\mathcal{I}_{0^{+}}^{\alpha ; \psi} F_{x}(s)\left(\varsigma_{2}\right)-\mathcal{I}_{0^{+}}^{\alpha ; \psi} F_{x}(s)\left(\varsigma_{1}\right)\right| \\
& \leq \frac{f^{*}}{\Gamma(\alpha+1)}\left[\left(\psi\left(\varsigma_{2}\right)-\psi\left(\varsigma_{1}\right)\right)^{\alpha}\right. \\
& \quad\left.+\left|\left(\psi\left(\varsigma_{2}\right)-\psi(0)\right)^{\alpha}-\left(\psi\left(\varsigma_{2}\right)-\psi\left(\varsigma_{1}\right)\right)^{\alpha}-\left(\psi\left(\varsigma_{1}\right)-\psi(0)\right)^{\alpha}\right|\right] .
\end{aligned}
$$

Thus the right-hand side of (28) (independently of the unknown variable $x$ ) approaches 0 as $\varsigma_{2} \rightarrow \varsigma_{1}$. Therefore $\mathcal{Q}_{1}$ is equicontinuous. Thus by the Arzelà-Ascoli theorem $\mathcal{Q}_{1}$ is relatively compact. 
Furthermore, it is easy to compute, utilizing (27), that $\mathcal{Q}_{2}$ is a contraction, and so Lemma 3.5(iii) holds. Therefore Lemma 3.5 is fulfilled, and thus a solution exists on $\mathbb{J}$ for the $\psi$ Hilfer FBVP describing the thermostat control model (3).

\section{The Ulam stability analysis}

In this section, we prove that the $\psi$-Hilfer FBVP describing the thermostat control model (3) is Ulam-Hyers $(\mathbb{U H H})$ stable, generalized Ulam-Hyers $(\mathbb{G} \mathbb{U H})$ stable, Ulam-HyersRassias $(\mathbb{U H} \mathbb{R})$ stable, and generalized Ulam-Hyers-Rassias $(\mathbb{G U H \mathbb { R }})$ stable.

Definition 4.1 ([38]) The $\psi$-Hilfer FBVP describing the thermostat control model (3) is said to be $\mathbb{U} \mathbb{H}$ stable if there exists $M_{f} \in \mathbb{R}^{+}$such that for any $\epsilon>0$ and for every solution $z \in \mathbb{E}$ of

$$
\left|{ }^{H} \mathfrak{D}_{0^{+}}^{\alpha, \rho ; \psi} z(\varsigma)-f\left(\varsigma, z(\theta \varsigma), \mathcal{I}_{0^{+}}^{q ; \psi} z(\varepsilon \varsigma)\right)\right| \leq \epsilon
$$

there is a solution $x \in \mathbb{E}$ of the $\psi$-Hilfer FBVP describing the thermostat control model (3) such that

$$
|z(\varsigma)-x(\varsigma)| \leq M_{f} \epsilon, \quad \varsigma \in \mathbb{J} .
$$

Definition 4.2 ([38]) The $\psi$-Hilfer FBVP describing the thermostat control model (3) is said to be $\mathbb{G} \mathbb{U H}$ stable if there exists $\mathcal{B} \in \mathcal{C}\left(\mathbb{R}^{+}, \mathbb{R}^{+}\right)$subject to $\mathcal{B}(0)=0$ such that, for every solution $z \in \mathbb{E}$ of

$$
\left|{ }^{H} \mathfrak{D}_{0^{+}}^{\alpha, \rho ; \psi} z(\varsigma)-f\left(\varsigma, z(\theta \varsigma), \mathcal{I}_{0^{+}}^{q ; \psi} z(\varepsilon \varsigma)\right)\right| \leq \epsilon \mathcal{B}(\varsigma)
$$

there is a solution $x \in \mathbb{E}$ of $\psi$-Hilfer FBVP describing the thermostat control model (3) such that

$$
|z(\varsigma)-x(\varsigma)| \leq \mathcal{B}(\epsilon), \quad \varsigma \in \mathbb{J} .
$$

Definition 4.3 ([38]) The $\psi$-Hilfer FBVP describing the thermostat control model (3) is said to be $\mathbb{U} \mathbb{H} \mathbb{R}$ stable by terms of $\mathcal{B} \in \mathcal{C}\left(\mathbb{J}, \mathbb{R}^{+}\right)$if there exists $M_{f, \mathcal{B}} \in \mathbb{R}^{+}$such that for each $\epsilon>0$ and for every solution $z \in \mathbb{E}$ of (31), there is a solution $x \in \mathbb{C}$ of the $\psi$-Hilfer FBVP describing the thermostat control model (3) such that

$$
|z(\varsigma)-x(\varsigma)| \leq M_{f, \mathcal{B}} \in \mathcal{B}(\varsigma), \quad \varsigma \in \mathbb{J} .
$$

Definition 4.4 ([38]) The $\psi$-Hilfer FBVP describing the thermostat control model (3) is said to be $\mathbb{G} \mathbb{U} \mathbb{R} \mathbb{R}$ stable by terms of $\mathcal{B} \in \mathcal{C}\left(\mathbb{J}, \mathbb{R}^{+}\right)$if there is $M_{f, \mathcal{B}} \in \mathbb{R}^{+}$such that for every solution $z \in \mathbb{E}$ of

$$
\left|{ }^{H} \mathfrak{D}_{0^{+}}^{\alpha, \rho ; \psi} z(\varsigma)-f\left(\varsigma, z(\theta \varsigma), \mathcal{I}_{0^{+}}^{q ; \psi} z(\varepsilon \varsigma)\right)\right| \leq \mathcal{B}(\varsigma),
$$

there is a solution $x \in \mathbb{E}$ of the $\psi$-Hilfer FBVP describing the thermostat control model (3) such that

$$
|z(\varsigma)-x(\varsigma)| \leq M_{f, \mathcal{B}} \mathcal{B}(\varsigma), \quad \varsigma \in \mathbb{J} .
$$


Remark 4.5 We easily see that:

$\left(a_{1}\right)$ Def. $4.1 \Rightarrow$ Def. 4.2;

$\left(a_{2}\right)$ Def. $4.3 \Rightarrow$ Def. 4.4;

$\left(a_{3}\right)$ Def. 4.3 for $\mathcal{B}(\varsigma)=1 \Rightarrow$ Def. 4.1.

Remark 4.6 $z \in \mathbb{E}$ is a solution of (29) if there exists $\mathrm{f} u \in \mathbb{E}$ (where $u$ depends on $z$ ) such that:

(i) $|v(\varsigma)| \leq \epsilon, \forall \varsigma \in \mathbb{J}$.

(ii) ${ }^{H} \mathfrak{D}_{0^{+}}^{\alpha, \rho ; \psi} z(\varsigma)=f\left(\varsigma, z(\theta \varsigma), \mathcal{I}_{0^{+}}^{q ; \psi} z(\varepsilon \varsigma)\right)+u(\varsigma), \varsigma \in \mathbb{J}$.

Remark $4.7 z \in \mathbb{E}$ is a solution of (31) if there exists $\mathrm{f} v \in \mathbb{E}$ (depending on $z$ ) such that:

(i) $|v(\varsigma)| \leq \epsilon \mathcal{B}(\varsigma), \forall \varsigma \in \mathbb{J}$.

(ii) ${ }^{H} \mathfrak{D}_{0^{+}}^{\alpha, \rho ; \psi} z(\varsigma)=f\left(\varsigma, z(\theta \varsigma), \mathcal{I}_{0^{+}}^{q ; \psi} z(\varepsilon \varsigma)\right)+v(\varsigma), \varsigma \in \mathbb{J}$.

Remark 4.8 There exist an increasing function $\mathcal{B} \in \mathcal{C}\left(\mathbb{J}, \mathbb{R}^{+}\right)$and a constant $n_{\mathcal{B}}>0$ such that for each $\varsigma \in \mathbb{J}$, we have the following integral inequality:

$$
\mathcal{I}_{0^{+}}^{\alpha ; \psi} \mathcal{B}(\varsigma) \leq n_{\mathcal{B}} \mathcal{B}(\varsigma)
$$

\subsection{The Ulam-Hyers stability}

First, we give the following lemma, which will be utilized in the arguments on $\mathbb{U H}$ and $\mathbb{G U H H}$ stability of problem (3).

Lemma 4.9 Let $\rho \in[0,1]$ and $\alpha \in(1,2]$, and let $z \in \mathbb{E}$ be the solution of (29). Then $z \in \mathbb{E}$ fulfills the integral inequality

$$
\left|z(\varsigma)-\mathcal{X}_{z}(\varsigma)-\mathcal{I}_{0^{+}}^{\alpha ; \psi} F_{z}(\varsigma)\right| \leq \Lambda(1,0) \epsilon
$$

where

$$
\begin{aligned}
\mathcal{X}_{z}(\varsigma)= & \frac{(\psi(\varsigma)-\psi(0))^{\gamma-1}}{\Omega \Gamma(\gamma)}\left[\Omega_{4}\left(A-\sum_{i=1}^{m} \omega_{i} \mathcal{I}_{0^{+}}^{\alpha-\beta_{i} ; \psi} F_{z}\left(\xi_{i}\right)\right)\right. \\
& \left.-\Omega_{2}\left(B-\sum_{j=1}^{n} \lambda_{j} \mathcal{I}_{0^{+}}^{\alpha-\mu_{j} ; \psi} F_{z}\left(\sigma_{j}\right)-\sum_{k=1}^{r} \delta_{k} \mathcal{I}_{0^{+}}^{\alpha ; \psi} F_{z}\left(\eta_{k}\right)\right)\right] \\
& +\frac{(\psi(\varsigma)-\psi(0))^{\gamma-2}}{\Omega \Gamma(\gamma-1)}\left[\Omega_{1}\left(B-\sum_{j=1}^{n} \lambda_{j} \mathcal{I}_{0^{+}}^{\alpha-\mu_{j} ; \psi} F_{z}\left(\sigma_{j}\right)-\sum_{k=1}^{r} \delta_{k} \mathcal{I}_{0^{+}}^{\alpha ; \psi} F_{z}\left(\eta_{k}\right)\right)\right. \\
& \left.-\Omega_{3}\left(A-\sum_{i=1}^{m} \omega_{i} \mathcal{I}_{0^{+}}^{\alpha-\beta_{i} ; \psi} F_{z}\left(\xi_{i}\right)\right)\right]
\end{aligned}
$$

with $\Omega_{1}, \Omega_{2}, \Omega_{3}, \Omega_{4}, \Omega$, and $\Lambda(\cdot, \cdot)$ given by (6), (7) (8), (9), (10), and (15), respectively.

Proof Let $z$ satisfy (29). By Remark 4.6(ii) and Lemma 2.8 we obtain

$$
\left\{\begin{array}{l}
{ }^{H} \mathfrak{D}_{0^{+}}^{\alpha, \rho ; \psi} z(\varsigma)=f\left(\varsigma, z(\theta \varsigma), \mathcal{I}_{0^{+}}^{q ; \psi} z(\varepsilon \varsigma)\right)+u(\varsigma), \quad \varsigma \in(0, T], \\
\sum_{i=1}^{m} \omega_{i}^{H} \mathfrak{D}_{0^{+}}^{\beta_{i}, \rho ; \psi} z\left(\xi_{i}\right)=A, \quad \sum_{j=1}^{n} \lambda_{j}{ }^{H} \mathfrak{D}_{0^{+}}^{\mu_{j}, \rho ; \psi} z\left(\sigma_{j}\right)+\sum_{k=1}^{r} \delta_{k} z\left(\eta_{k}\right)=B .
\end{array}\right.
$$


Then the solution of (39) can be written in the form

$$
\begin{aligned}
z(\varsigma)= & \mathcal{I}_{0^{+}}^{\alpha ; \psi} F_{z}(\varsigma)+\frac{(\psi(\varsigma)-\psi(0))^{\gamma-1}}{\Omega \Gamma(\gamma)}\left[\Omega_{4}\left(A-\sum_{i=1}^{m} \omega_{i} \mathcal{I}_{0^{+}}^{\alpha-\beta_{i} ; \psi} F_{z}\left(\xi_{i}\right)\right)\right. \\
& \left.-\Omega_{2}\left(B-\sum_{j=1}^{n} \lambda_{j} \mathcal{I}_{0^{+}}^{\alpha-\mu_{j} ; \psi} F_{z}\left(\sigma_{j}\right)-\sum_{k=1}^{r} \delta_{k} \mathcal{I}_{0^{+}}^{\alpha ; \psi} F_{z}\left(\eta_{k}\right)\right)\right] \\
& +\frac{(\psi(\varsigma)-\psi(0))^{\gamma-2}}{\Omega \Gamma(\gamma-1)}\left[\Omega_{1}\left(B-\sum_{j=1}^{n} \lambda_{j} \mathcal{I}_{0^{+}}^{\alpha-\mu_{j} ; \psi} F_{z}\left(\sigma_{j}\right)-\sum_{k=1}^{r} \delta_{k} \mathcal{I}_{0^{+}}^{\alpha ; \psi} F_{z}\left(\eta_{k}\right)\right)\right. \\
& \left.-\Omega_{3}\left(A-\sum_{i=1}^{m} \omega_{i} \mathcal{I}_{0^{+}}^{\alpha-\beta_{i} ; \psi} F_{z}\left(\xi_{i}\right)\right)\right] \\
& +\mathcal{I}_{0^{+}}^{\alpha ; \psi} u(\varsigma)+\frac{(\psi(\varsigma)-\psi(0))^{\gamma-1}}{\Omega \Gamma(\gamma)}\left[\Omega_{2}\left(\sum_{j=1}^{n} \lambda_{j} \mathcal{I}_{0^{+}}^{\alpha-\mu_{j} ; \psi} u\left(\sigma_{j}\right)+\sum_{k=1}^{r} \delta_{k} \mathcal{I}_{0^{+}}^{\alpha ; \psi} u\left(\eta_{k}\right)\right)\right. \\
& \left.-\Omega_{4} \sum_{i=1}^{m} \omega_{i} \mathcal{I}_{0^{+}}^{\alpha-\beta_{i} ; \psi} u\left(\xi_{i}\right)\right]+\frac{(\psi(\varsigma)-\psi(0))^{\gamma-2}}{\Omega \Gamma(\gamma-1)}\left[\Omega_{3} \sum_{i=1}^{m} \omega_{i} \mathcal{I}_{0^{+}}^{\alpha-\beta_{i} ; \psi} u\left(\xi_{i}\right)\right. \\
& \left.-\Omega_{1}\left(\sum_{j=1}^{n} \lambda_{j} \mathcal{I}_{0^{+}}^{\alpha-\mu_{j} ; \psi} u\left(\sigma_{j}\right)+\sum_{k=1}^{r} \delta_{k} \mathcal{I}_{0^{+}}^{\alpha ; \psi} u\left(\eta_{k}\right)\right)\right] .
\end{aligned}
$$

Remark 4.6(i) implies that

$$
\begin{aligned}
\mid z(\varsigma) & -\mathcal{X}_{z}(\varsigma)-\mathcal{I}_{0^{+}}^{\alpha ; \psi} F_{z}(\varsigma) \mid \\
= & \mathcal{I}_{0^{+}}^{\alpha ; \psi} u(\varsigma)+\frac{(\psi(\varsigma)-\psi(0))^{\gamma-1}}{\Omega \Gamma(\gamma)}\left[\Omega_{2}\left(\sum_{j=1}^{n} \lambda_{j} \mathcal{I}_{0^{+}}^{\alpha-\mu_{j} ; \psi} u\left(\sigma_{j}\right)+\sum_{k=1}^{r} \delta_{k} \mathcal{I}_{0^{+}}^{\alpha ; \psi} u\left(\eta_{k}\right)\right)\right. \\
& \left.-\Omega_{4} \sum_{i=1}^{m} \omega_{i} \mathcal{I}_{0^{+}}^{\alpha-\beta_{i} ; \psi} u\left(\xi_{i}\right)\right]+\frac{(\psi(\varsigma)-\psi(0))^{\gamma-2}}{\Omega \Gamma(\gamma-1)}\left[\Omega_{3} \sum_{i=1}^{m} \omega_{i} \mathcal{I}_{0^{+}}^{\alpha-\beta_{i} ; \psi} u\left(\xi_{i}\right)\right. \\
& \left.-\Omega_{1}\left(\sum_{j=1}^{n} \lambda_{j} \mathcal{I}_{0^{+}}^{\alpha-\mu_{j} ; \psi} u\left(\sigma_{j}\right)+\sum_{k=1}^{r} \delta_{k} \mathcal{I}_{0^{+}}^{\alpha ; \psi} u\left(\eta_{k}\right)\right)\right] \mid \\
\leq & \left\{\Psi_{1}(T, \alpha)+\frac{1}{|\Omega|}\left(\left|\Omega_{4}\right| \Psi_{1}(T, \gamma-1)+\left|\Omega_{3}\right| \Psi_{1}(T, \gamma-2)\right) \sum_{i=1}^{m}\left|\omega_{i}\right| \Psi_{1}\left(\xi_{i}, \alpha-\beta_{i}\right)\right. \\
& +\frac{1}{|\Omega|}\left|\Omega_{2}\right|\left(\Psi_{1}(T, \gamma-1)+\left|\Omega_{1}\right| \Psi_{1}(T, \gamma-2)\right) \sum_{j=1}^{n}\left|\lambda_{j}\right| \Psi_{1}\left(\sigma_{j}, \alpha-\mu_{j}\right) \\
& \left.+\frac{1}{|\Omega|}\left(\left|\Omega_{2}\right| \Psi_{1}(T, \gamma-1)+\left|\Omega_{1}\right| \Psi_{1}(T, \gamma-2)\right) \sum_{k=1}^{r}\left|\delta_{k}\right| \Psi_{1}\left(\eta_{k}, \alpha\right)\right\} \epsilon \\
= & \left\{\Psi_{1}(T, \alpha)+\Psi_{2}\left(\Omega_{3}, \Omega_{4}\right) \sum_{i=1}^{m}\left|\omega_{i}\right| \Psi_{1}\left(\xi_{i}, \alpha-\beta_{i}\right)\right. \\
& \left.+\Psi_{2}\left(\Omega_{1}, \Omega_{2}\right)\left(\sum_{j=1}^{n}\left|\lambda_{j}\right| \Psi_{1}\left(\sigma_{j}, \alpha-\mu_{j}\right)+\sum_{k=1}^{r}\left|\delta_{k}\right| \Psi_{1}\left(\eta_{k}, \alpha\right)\right)\right\}
\end{aligned}
$$




$$
=\Lambda(1,0) \epsilon .
$$

Inequality (37) is achieved.

Next, we prove the $\mathbb{U H}$ and $\mathbb{G} \mathbb{U H}$ stability of solution to problem (3).

Theorem 4.10 Let $f: \mathbb{J} \times \mathbb{R}^{2} \rightarrow \mathbb{R}$ be continuous, and let $\left(H_{1}\right)$ be satisfied subject to

$$
L_{1} \Psi_{1}(T, \alpha)+L_{2} \Psi_{1}(T, q+\alpha)<1
$$

Then the $\psi$-Hilfer FBVP describing the thermostat control model (3) is $\mathbb{U} \mathbb{H}$ and $\mathbb{G} \mathbb{U H}$ stable in $\mathbb{E}$.

Proof Let $z \in \mathbb{E}$ satisfy (29), and let $x \in \mathbb{E}$ be a unique solution of the $\psi$-Hilfer FBVP describing the thermostat control model (3) with nonlocal conditions of the form

$$
\sum_{i=1}^{m} \omega_{i}^{H} \mathfrak{D}_{0^{+}}^{\beta_{i}, \rho ; \psi} x\left(\xi_{i}\right)=A \quad \text { and } \quad \sum_{j=1}^{n} \lambda_{j}{ }^{H} \mathfrak{D}_{0^{+}}^{\mu_{j}, \rho ; \psi} x\left(\sigma_{j}\right)+\sum_{k=1}^{r} \delta_{k} x\left(\eta_{k}\right)=B
$$

By using Lemma 2.8 we have

$$
x(\varsigma)=\mathcal{X}_{x}(\varsigma)+\mathcal{I}_{0^{+}}^{\alpha ; \psi} F_{x}(\varsigma)
$$

where

$$
\begin{aligned}
\mathcal{X}_{x}(\varsigma)= & \frac{(\psi(\varsigma)-\psi(0))^{\gamma-1}}{\Omega \Gamma(\gamma)}\left[\Omega_{4}\left(A-\sum_{i=1}^{m} \omega_{i} \mathcal{I}_{0^{+}}^{\alpha-\beta_{i} ; \psi} F_{x}\left(\xi_{i}\right)\right)\right. \\
& \left.-\Omega_{2}\left(B-\sum_{j=1}^{n} \lambda_{j} \mathcal{I}_{0^{+}}^{\alpha-\mu_{j} ; \psi} F_{x}\left(\sigma_{j}\right)-\sum_{k=1}^{r} \delta_{k} \mathcal{I}_{0^{+}}^{\alpha ; \psi} F_{x}\left(\eta_{k}\right)\right)\right] \\
& +\frac{(\psi(\varsigma)-\psi(0))^{\gamma-2}}{\Omega \Gamma(\gamma-1)}\left[\Omega_{1}\left(B-\sum_{j=1}^{n} \lambda_{j} \mathcal{I}_{0^{+}}^{\alpha-\mu_{j} ; \psi} F_{x}\left(\sigma_{j}\right)-\sum_{k=1}^{r} \delta_{k} \mathcal{I}_{0^{+}}^{\alpha ; \psi} F_{x}\left(\eta_{k}\right)\right)\right. \\
& \left.-\Omega_{3}\left(A-\sum_{i=1}^{m} \omega_{i} \mathcal{I}_{0^{+}}^{\alpha-\beta_{i} ; \psi} F_{x}\left(\xi_{i}\right)\right)\right] .
\end{aligned}
$$

On other hand, if ${ }^{H} \mathfrak{D}_{0^{+}}^{\beta_{i}, \rho ; \psi} x\left(\xi_{i}\right)={ }^{H} \mathfrak{D}_{0^{+}}^{\beta_{i}, \rho ; \psi} z\left(\xi_{i}\right),{ }^{H} \mathfrak{D}_{0^{+}}^{\mu_{j}, \rho ; \psi} x\left(\sigma_{j}\right)={ }^{H} \mathfrak{D}_{0^{+}}^{\mu_{j}, \rho ; \psi} z\left(\sigma_{j}\right)$, and $x\left(\eta_{k}\right)=$ $z\left(\eta_{k}\right)$, then $\mathcal{X}_{x}(\varsigma)=\mathcal{X}_{z}(\varsigma)$. By applying the triangle inequality $|x+y| \leq|x|+|y|$ and Lemma 4.9, for $\varsigma \in \mathbb{J}$, we obtain

$$
\begin{aligned}
& |z(\varsigma)-x(\varsigma)| \\
& \quad=\left|z(\varsigma)-\mathcal{X}_{x}(\varsigma)-\mathcal{I}_{0^{+}}^{\alpha ; \psi} F_{x}(\varsigma)\right| \\
& \quad \leq\left|z(\varsigma)-\mathcal{X}_{z}(\varsigma)-\mathcal{I}_{0^{+}}^{\alpha ; \psi} F_{z}(\varsigma)\right|+\mathcal{I}_{0^{+}}^{\alpha ; \psi}\left|F_{z}(s)-F_{x}(s)\right|(\varsigma)+\left|\mathcal{X}_{z}(\varsigma)-\mathcal{X}_{x}(\varsigma)\right| \\
& \quad \leq \Lambda(1,0) \epsilon+\left(L_{1} \Psi_{1}(T, \alpha)+L_{2} \Psi_{1}(T, q+\alpha)\right)|z(\varsigma)-x(\varsigma)|,
\end{aligned}
$$


which implies that

$$
|z(\varsigma)-x(\varsigma)| \leq \frac{\Lambda(1,0)}{1-\left(L_{1} \Psi_{1}(T, \alpha)+L_{2} \Psi_{1}(T, q+\alpha)\right)} \epsilon .
$$

By setting

$$
M_{f}=\frac{\Lambda(1,0)}{1-\left(L_{1} \Psi_{1}(T, \alpha)+L_{2} \Psi_{1}(T, q+\alpha)\right)}
$$

we get $|z(\varsigma)-x(\varsigma)| \leq M_{f} \epsilon$. Therefore problem (3) is $\mathbb{U H}$ stable. Moreover, if we take $\mathcal{B}(\epsilon)=$ $M_{f} \in$ with $\mathcal{B}(0)=0$, then the $\psi$-Hilfer FBVP describing the thermostat control model (3) is $\mathbb{G U H H}$ stable.

\subsection{The Ulam-Hyers-Rassias stability}

This lemma will is helpful in the arguments on $\mathbb{U} \mathbb{R} \mathbb{R}$ and $\mathbb{G} U \mathbb{H} \mathbb{R}$ stability of our results.

Lemma 4.11 Let $\rho \in[0,1]$ and $\alpha \in(1,2]$, and let $z \in \mathbb{E}$ be the solution of (31). Then $z \in \mathbb{E}$ fulfills the integral inequality

$$
\left|z(\varsigma)-\mathcal{X}_{z}(\varsigma)-\mathcal{I}_{0^{+}}^{\alpha ; \psi} F_{z}(\varsigma)\right| \leq \Theta \epsilon n_{\mathcal{B}} \mathcal{B}(\varsigma),
$$

where

$$
\Theta=1+\Psi_{2}\left(\Omega_{1}, \Omega_{2}\right)\left(\sum_{j=1}^{n}\left|\lambda_{j}\right|+\sum_{k=1}^{r}\left|\delta_{k}\right|\right)+\Psi_{2}\left(\Omega_{3}, \Omega_{4}\right) \sum_{i=1}^{m}\left|\omega_{i}\right|,
$$

and $\mathcal{X}_{z}(\varsigma)$ is defined by (38).

Proof Let $z$ satisfy (31). By Remark 4.7(ii) and Lemma 2.8 the solution of the problem

$$
\left\{\begin{array}{l}
{ }^{H} \mathfrak{D}_{0^{+}}^{\alpha, \rho ; \psi} z(\varsigma)=f\left(\varsigma, z(\theta \varsigma), \mathcal{I}_{0^{+}}^{q ; \psi} z(\varepsilon \varsigma)\right)+v(\varsigma), \quad \varsigma \in(0, T], \\
\sum_{i=1}^{m} \omega_{i}^{H} \mathfrak{D}_{0^{+}}^{\beta_{i}, \rho ; \psi} z\left(\xi_{i}\right)=A, \quad \sum_{j=1}^{n} \lambda_{j}^{H} \mathfrak{D}_{0^{+}}^{\mu_{j}, \rho ; \psi} z\left(\sigma_{j}\right)+\sum_{k=1}^{r} \delta_{k} z\left(\eta_{k}\right)=B,
\end{array}\right.
$$

can be written in the following form:

$$
\begin{aligned}
z(\varsigma)= & \mathcal{I}_{0^{+}}^{\alpha ; \psi} F_{z}(\varsigma)+\frac{(\psi(\varsigma)-\psi(0))^{\gamma-1}}{\Omega \Gamma(\gamma)}\left[\Omega_{4}\left(A-\sum_{i=1}^{m} \omega_{i} \mathcal{I}_{0^{+}}^{\alpha-\beta_{i} ; \psi} F_{z}\left(\xi_{i}\right)\right)\right. \\
& \left.-\Omega_{2}\left(B-\sum_{j=1}^{n} \lambda_{j} \mathcal{I}_{0^{+}}^{\alpha-\mu_{j} ; \psi} F_{z}\left(\sigma_{j}\right)-\sum_{k=1}^{r} \delta_{k} \mathcal{I}_{0^{+}}^{\alpha ; \psi} F_{z}\left(\eta_{k}\right)\right)\right] \\
& +\frac{(\psi(\varsigma)-\psi(0))^{\gamma-2}}{\Omega \Gamma(\gamma-1)}\left[\Omega_{1}\left(B-\sum_{j=1}^{n} \lambda_{j} \mathcal{I}_{0^{+}}^{\alpha-\mu_{j} ; \psi} F_{z}\left(\sigma_{j}\right)-\sum_{k=1}^{r} \delta_{k} \mathcal{I}_{0^{+}}^{\alpha ; \psi} F_{z}\left(\eta_{k}\right)\right)\right. \\
& \left.-\Omega_{3}\left(A-\sum_{i=1}^{m} \omega_{i} \mathcal{I}_{0^{+}}^{\alpha-\beta_{i} ; \psi} F_{z}\left(\xi_{i}\right)\right)\right] \\
& +\mathcal{I}_{0^{+}}^{\alpha ; \psi} \nu(\varsigma)+\frac{(\psi(\varsigma)-\psi(0))^{\gamma-1}}{\Omega \Gamma(\gamma)}\left[\Omega_{2}\left(\sum_{j=1}^{n} \lambda_{j} \mathcal{I}_{0^{+}}^{\alpha-\mu_{j} ; \psi} \nu\left(\sigma_{j}\right)+\sum_{k=1}^{r} \delta_{k} \mathcal{I}_{0^{+}}^{\alpha ; \psi} v\left(\eta_{k}\right)\right)\right.
\end{aligned}
$$




$$
\begin{aligned}
& \left.-\Omega_{4} \sum_{i=1}^{m} \omega_{i} \mathcal{I}_{0^{+}}^{\alpha-\beta_{i} ; \psi} v\left(\xi_{i}\right)\right]+\frac{(\psi(\varsigma)-\psi(0))^{\gamma-2}}{\Omega \Gamma(\gamma-1)}\left[\Omega_{3} \sum_{i=1}^{m} \omega_{i} \mathcal{I}_{0^{+}}^{\alpha-\beta_{i} ; \psi} \nu\left(\xi_{i}\right)\right. \\
& \left.-\Omega_{1}\left(\sum_{j=1}^{n} \lambda_{j} \mathcal{I}_{0^{+}}^{\alpha-\mu_{j} ; \psi} v\left(\sigma_{j}\right)+\sum_{k=1}^{r} \delta_{k} \mathcal{I}_{0^{+}}^{\alpha ; \psi} u v\left(\eta_{k}\right)\right)\right] .
\end{aligned}
$$

From Remarks 4.7(i) and 4.8 we obtain the following estimate:

$$
\begin{aligned}
\mid z(\varsigma) & -\mathcal{X}_{z}(\varsigma)-\mathcal{I}_{0^{+}}^{\alpha ; \psi} F_{z}(\varsigma) \mid \\
= & \mid \mathcal{I}_{0^{+}}^{\alpha ; \psi} \nu(\varsigma)+\frac{(\psi(\varsigma)-\psi(0))^{\gamma-1}}{\Omega \Gamma(\gamma)}\left[\Omega_{2}\left(\sum_{j=1}^{n} \lambda_{j} \mathcal{I}_{0^{+}}^{\alpha-\mu_{j} ; \psi} \nu\left(\sigma_{j}\right)+\sum_{k=1}^{r} \delta_{k} \mathcal{I}_{0^{+}}^{\alpha ; \psi} \nu\left(\eta_{k}\right)\right)\right. \\
& \left.-\Omega_{4} \sum_{i=1}^{m} \omega_{i} \mathcal{I}_{0^{+}}^{\alpha-\beta_{i} ; \psi} v\left(\xi_{i}\right)\right]+\frac{(\psi(\varsigma)-\psi(0))^{\gamma-2}}{\Omega \Gamma(\gamma-1)}\left[\Omega_{3} \sum_{i=1}^{m} \omega_{i} \mathcal{I}_{0^{+}}^{\alpha-\beta_{i} ; \psi} \nu\left(\xi_{i}\right)\right. \\
& \left.-\Omega_{1}\left(\sum_{j=1}^{n} \lambda_{j} \mathcal{I}_{0^{+}}^{\alpha-\mu_{j} ; \psi} v\left(\sigma_{j}\right)+\sum_{k=1}^{r} \delta_{k} \mathcal{I}_{0^{+}}^{\alpha ; \psi} \nu\left(\eta_{k}\right)\right)\right] \mid \\
\leq & \left\{1+\frac{\Psi_{1}(T, \gamma-1)}{|\Omega|}\left[\left|\Omega_{2}\right|\left(\sum_{j=1}^{n}\left|\lambda_{j}\right|+\sum_{k=1}^{r}\left|\delta_{k}\right|\right)+\left|\Omega_{4}\right| \sum_{i=1}^{m}\left|\omega_{i}\right|\right]\right. \\
& \left.+\frac{\Psi_{1}(T, \gamma-2)}{|\Omega|}\left[\left|\Omega_{3}\right| \sum_{i=1}^{m}\left|\omega_{i}\right|+\left|\Omega_{1}\right|\left(\sum_{j=1}^{n}\left|\lambda_{j}\right|+\sum_{k=1}^{r}\left|\delta_{k}\right|\right)\right]\right\} \in n_{\mathcal{B}} \mathcal{B}(\varsigma) \\
= & \Theta \epsilon n_{\mathcal{B}} \mathcal{B}(\varsigma),
\end{aligned}
$$

from which we get (41).

Next, we check $\mathbb{U} \mathbb{R}$ and $\mathbb{G} \mathbb{U} \mathbb{R}$ stability of solution to problem (3).

Theorem 4.12 Let $f: \mathbb{J} \times \mathbb{R}^{2} \rightarrow \mathbb{R}$ be a continuous mapping under the assumption $\left(H_{1}\right)$, and let (36) be satisfied. If $\left(L_{1} \Psi_{1}(T, \alpha)+L_{2} \Psi_{1}(T, q+\alpha)\right)<1$, then the $\psi$-Hilfer FBVP describing the thermostat control model (3) is $\mathbb{U} \mathbb{R} \mathbb{R}$ and $\mathbb{G} U \mathbb{H} \mathbb{R}$ stable in $\mathbb{E}$.

Proof Let $z \in \mathbb{E}$ satisfy (31), and let $x$ be a unique solution of (3). By Lemma 4.11 we get

$$
x(\varsigma)=\mathcal{X}_{x}(\varsigma)+\mathcal{I}_{0^{+}}^{\alpha ; \psi} F_{x}(\varsigma)
$$

where $\mathcal{X}_{x}(\varsigma)$ is given by (40). Similarly, if ${ }^{H} \mathfrak{D}_{0^{+}}^{\beta_{i}, \rho ; \psi} x\left(\xi_{i}\right)={ }^{H} \mathfrak{D}_{0^{+}}^{\beta_{i}, \rho ; \psi} z\left(\xi_{i}\right),{ }^{H} \mathfrak{D}_{0^{+}}^{\mu_{j}, \rho ; \psi} x\left(\sigma_{j}\right)=$ ${ }^{H} \mathfrak{D}_{0^{+}}^{\mu_{j}, \rho ; \psi} z\left(\sigma_{j}\right)$, and $x\left(\eta_{k}\right)=z\left(\eta_{k}\right)$, then $\mathcal{X}_{x}(\varsigma)=\mathcal{X}_{z}(\varsigma)$. Applying the triangle inequality with Lemma 4.11, for $\varsigma \in \mathbb{J}$, we estimate

$$
\begin{aligned}
|z(\varsigma)-x(\varsigma)| & =\left|z(\varsigma)-\mathcal{X}_{x}(\varsigma)-\mathcal{I}_{0^{+}}^{\alpha ; \psi} F_{x}(\varsigma)\right| \\
& \leq\left|z(\varsigma)-\mathcal{X}_{z}(\varsigma)-\mathcal{I}_{0^{+}}^{\alpha ; \psi} F_{z}(\varsigma)\right|+\mathcal{I}_{0^{+}}^{\alpha ; \psi}\left|F_{z}(s)-F_{x}(s)\right|(\varsigma)+\left|\mathcal{X}_{z}(\varsigma)-\mathcal{X}_{x}(\varsigma)\right| \\
& \leq \Theta \epsilon n_{\mathcal{B}} \mathcal{B}(\varsigma)+\left(L_{1} \Psi_{1}(T, \alpha)+L_{2} \Psi_{1}(T, q+\alpha)\right)|z(\varsigma)-x(\varsigma)|
\end{aligned}
$$


where $\Theta$ and $\Psi_{1}(\cdot, \cdot)$ are defined by (42) and (13), respectively. Then

$$
|z(\varsigma)-x(\varsigma)| \leq \frac{\Theta n_{\mathcal{B}}}{1-\left(L_{1} \Psi_{1}(T, \alpha)+L_{2} \Psi_{1}(T, q+\alpha)\right)} \mathcal{B}(\varsigma) \epsilon .
$$

By setting

$$
M_{f, \mathcal{B}}=\frac{\Theta n_{\mathcal{B}}}{1-\left(L_{1} \Psi_{1}(T, \alpha)+L_{2} \Psi_{1}(T, q+\alpha)\right)}
$$

we get the estimate

$$
|z(\varsigma)-x(\varsigma)| \leq M_{f, \mathcal{B}} \mathcal{B}(\varsigma) \epsilon
$$

This proves that the $\psi$-Hilfer FBVP describing the thermostat control model (3) is $\mathbb{U H} \mathbb{R}$ stable. Furthermore, taking $\epsilon=1$ in (44) with $\mathcal{B}(0)=0$, the $\psi$-Hilfer FBVP describing the thermostat control model (3) is $\mathbb{G} \mathbb{U H} \mathbb{R}$ stable.

\section{Example}

In this section, we provide some examples compatible to the exactitude and applicability of our main results.

Example 5.1 Consider the $\psi$-Hilfer FBVP describing the thermostat control model

$$
\left\{\begin{array}{l}
H \mathfrak{D}_{0^{+}}^{\frac{3}{2}, \frac{1}{3} ; e^{\frac{\sqrt{\varsigma}}{2}}} x(\varsigma)=f\left(\varsigma, x(\theta \varsigma), \mathcal{I}_{0^{+}}^{\sqrt{\pi} ; e^{\frac{\sqrt{\zeta}}{2}}} x(\varepsilon \varsigma)\right), \quad \varsigma \in(0,3 / 2], \\
\sum_{i=1}^{m}\left(\frac{\tan ^{-1}(i)}{(i+1)^{2}}\right)^{H} \mathfrak{D}_{0^{+}}^{\frac{\pi}{2 i+1}, \frac{1}{3} ; e^{\frac{\sqrt{\varsigma}}{2}}} x\left(\frac{i}{i+1}\right)=\frac{\sqrt{3}}{2}, \\
\sum_{j=1}^{n}\left(\sin \left(\frac{\pi j}{j+1}\right)\right)^{H} \mathfrak{D}_{0^{+}}^{\frac{\sqrt{j}}{j+1}, \frac{1}{3} ; e^{\frac{\sqrt{\zeta}}{2}}} x\left(\frac{j+1}{2 j+3}\right)+\sum_{k=1}^{r}\left(e^{-\sqrt{k \pi}}\right) x\left(\frac{\sqrt{k}}{k+1}\right)=-\frac{1}{3} .
\end{array}\right.
$$

Here $\alpha=3 / 2, q=\sqrt{\pi}, \rho=1 / 3, \psi(\varsigma)=\exp (\sqrt{\varsigma} / 2), T=3 / 2, m=2, n=2, r=2, \beta_{i}=\pi /(2 i+$ 1), $\mu_{j}=\sqrt{j} /(j+1), \omega_{i}=\left(\tan ^{-1}(i)\right) /\left((i+1)^{2}\right), \lambda_{j}=\sin ((\pi j) /(j+1)), \delta_{k}=\exp (-\sqrt{k \pi}), \xi_{i}=i /(i+$ $1), \sigma_{j}=(j+1) /(2 j+3), \eta_{k}=\sqrt{k} /(k+1), i=j=k=1,2, A=\sqrt{3} / 2$, and $B=-1 / 3$. From this information we can calculate that $\Omega_{1} \approx 0.2318892799, \Omega_{2} \approx-0.2621694875, \Omega_{3} \approx$ $0.2107768913, \Omega_{4} \approx 0.1675500424$, and $\Omega \approx 0.09411232825 \neq 0$.

(i) Consider the function

$$
\begin{aligned}
& f\left(\varsigma, x(0.2 \varsigma), \mathcal{I}_{0^{+}}^{\sqrt{\pi} ; e^{\frac{\sqrt{\varsigma}}{2}}} x(0.5 \varsigma)\right) \\
& \quad=\frac{\varsigma}{\varsigma+1}+\frac{2 \varsigma+1}{2-\cos ^{2} \pi \varsigma} \cdot \frac{|x(0.2 \varsigma)|}{4+|x(0.2 \varsigma)|}+\frac{(4 \varsigma-1)^{2}}{4} \cdot \frac{\left|\mathcal{I}_{0^{+}}^{\sqrt{\pi} ; e^{\frac{\sqrt{\varsigma}}{2}}} x(0.5 \varsigma)\right|}{25+\left|\mathcal{I}_{0^{+}}^{\sqrt{\pi} ; e^{\frac{\sqrt{\varsigma}}{2}}} x(0.5 \varsigma)\right|}
\end{aligned}
$$

with $\theta=1 / 5$ and $\varepsilon=1 / 2$. For $x_{i}, y_{i} \in \mathbb{R}, i=1,2$, and $\varsigma \in[0,3 / 2]$, we can find that

$$
\left|f\left(\varsigma, x_{1}, y_{1}\right)-f\left(\varsigma, x_{2}, y_{2}\right)\right| \leq \frac{1}{2}\left|x_{1}-x_{2}\right|+\frac{1}{4}\left|y_{1}-y_{2}\right| .
$$


Assumption $\left(H_{1}\right)$ is satisfied with $L_{1}=\frac{1}{2}$ and $L_{2}=\frac{1}{4}$. Hence

$$
\Lambda(1 / 2,1 / 4) \approx 0.7369944526<1 \text {. }
$$

All assumptions of Theorem 3.2 are fulfilled; thus the $\psi$-Hilfer FBVP describing the thermostat control model (45) has exactly one solution on $[0,3 / 2]$. Further,

$$
M_{f}=\frac{\Lambda(1,0)}{1-\left(L_{1} \Psi_{1}(T, \alpha)+L_{2} \Psi_{1}(T, q+\alpha)\right)} \approx 1.066442242>0
$$

By Theorem 4.10 the $\psi$-Hilfer FBVP describing the thermostat control model (45) is both $\mathbb{U H}$ and $\mathbb{G} U \mathbb{H}$ stable on $[0,3 / 2]$. Setting $\mathcal{B}(\varsigma)=\psi(\varsigma)-\psi(0)$, by Proposition 2.5(i) we easily compute that

$$
\mathcal{I}_{0^{+}}^{\alpha ; \psi} \mathcal{B}(\varsigma)=\frac{1}{\Gamma\left(\frac{7}{2}\right)}(\psi(\varsigma)-\psi(0))^{\frac{5}{2}} \Theta(\varsigma) \leq \frac{8\left(e^{\frac{\sqrt{6}}{4}}-1\right)^{\frac{5}{2}}}{15 \sqrt{\pi}} \Theta(\varsigma)
$$

Then inequality (36) is satisfied with $n_{\Theta}=\frac{8\left(e^{\frac{\sqrt{6}}{4}}-1\right)^{\frac{5}{2}}}{15 \sqrt{\pi}}>0$ and $\Theta \approx 2.901034837$. It follows that

$$
M_{f, \mathcal{B}}=\frac{\Theta n_{\mathcal{B}}}{1-\left(L_{1} \Psi_{1}(T, \alpha)+L_{2} \Psi_{1}(T, q+\alpha)\right)} \approx 0.4142937053>0 .
$$

Therefore by Theorem 4.12 the $\psi$-Hilfer FBVP describing the thermostat control model (45) is both $\mathbb{U} \mathbb{R} \mathbb{R}$ and $\mathbb{G} \mathbb{U} \mathbb{R}$ stable on $[0,3 / 2]$.

(ii) Consider the function

$$
\begin{array}{r}
f\left(\varsigma, x(\sqrt{2} \varsigma / 2), \mathcal{I}_{0^{+}}^{\sqrt{\pi} ; e^{\frac{\sqrt{\varsigma}}{2}}} x(\sqrt{3} \varsigma / 2)\right) \\
=\frac{\sqrt{2} e^{-\varsigma}}{(\varsigma+2)^{2}}+\frac{\sqrt{2 \varsigma+1}}{\varsigma+5} \cdot \sin ^{-1}|x(\sqrt{2} \varsigma / 2)| \\
\quad+\frac{4 \cos ^{2}(\pi \varsigma)}{e^{\varsigma}+1} \cdot \frac{\left|\mathcal{I}_{0^{+}}^{\sqrt{\pi} ; e^{\frac{\sqrt{\varsigma}}{2}}} x(\sqrt{3} \varsigma / 2)\right|}{4+\left|\mathcal{I}_{0^{+}}^{\sqrt{\pi} ; e^{\frac{\sqrt{5}}{2}}} x(\sqrt{3} \varsigma / 2)\right|}
\end{array}
$$

with $\theta=\sqrt{2} / 2$ and $\varepsilon=\sqrt{3} / 2$. For $x_{i}, y_{i} \in \mathbb{R}, i=1,2$, and $\varsigma \in[0,3 / 2]$, we can find that

$$
\begin{aligned}
& \left|f\left(\varsigma, x_{1}, y_{1}\right)-f\left(\varsigma, x_{2}, y_{2}\right)\right| \\
& \quad \leq \frac{\sqrt{2 \varsigma+1}}{\varsigma+5}\left|x_{1}-x_{2}\right|+\frac{\cos ^{2}(\pi \varsigma)}{e^{\varsigma}+1}\left|y_{1}-y_{2}\right| \leq \frac{2}{5}\left|x_{1}-x_{2}\right|+\frac{1}{2}\left|y_{1}-y_{2}\right| .
\end{aligned}
$$

This means that assumption $\left(H_{1}\right)$ is satisfied with $L_{1}=2 / 5, L_{2}=1 / 2$, and $\Lambda(2 / 5,1 / 2) \approx$ $0.6261447320<1$. Therefore, for any $x, y \in \mathbb{R}$ and $\varsigma \in[0,3 / 2]$, we can estimate

$$
|f(\varsigma, x, y)| \leq \frac{\sqrt{2} e^{-\varsigma}}{(\varsigma+2)^{2}}+\frac{\sqrt{2 \varsigma+1}}{\varsigma+5}|x|+\frac{\cos ^{2}(\pi \varsigma)}{e^{\varsigma}+1}|y|
$$


Assumption $\left(H_{2}\right)$ is also valid with $k_{1}=\left(\sqrt{2} e^{-\varsigma}\right) /\left((\varsigma+2)^{2}\right), k_{2}(\varsigma)=(\sqrt{2 \varsigma+1}) /(\varsigma+5)$, $k_{3}(\varsigma)=\left(\cos ^{2}(\pi \varsigma)\right) /\left(e^{\varsigma}+1\right)$, and $k_{1}^{*}=\sqrt{2} / 4, k_{2}^{*}=2 / 5 k_{3}^{*}=1 / 2$. Therefore the assumptions of Theorem 3.4 are fulfilled, and thus the $\psi$-Hilfer FBVP describing the thermostat control model (45) has at least one solution on [0,3/2]. Moreover, we get

$$
M_{f}=\frac{\Lambda(1,0)}{1-\left(L_{1} \Psi_{1}(T, \alpha)+L_{2} \Psi_{1}(T, q+\alpha)\right)} \approx 0.8546613342>0 .
$$

Therefore by Theorem 4.10 problem (45) is both $\mathbb{U H}$ and $\mathbb{G} \mathbb{U H}$ stable on $[0,3 / 2]$. Furthermore, if we set $\mathcal{B}(\varsigma)=(\psi(\varsigma)-\psi(0))^{3 / 2}$, then by Proposition $2.5(\mathrm{i})$ we easily compute that

$$
\mathcal{I}_{0^{+}}^{\alpha ; \psi} \mathcal{B}(\varsigma)=\frac{\Gamma\left(\frac{5}{2}\right)}{\Gamma\left(\frac{5}{2}+\alpha\right)}(\psi(\varsigma)-\psi(0))^{\frac{3}{2}+\alpha} \Theta(\varsigma) \leq \frac{\left(e^{\frac{\sqrt{6}}{4}}-1\right)^{3}}{8 \sqrt{\pi}} \Theta(\varsigma)
$$

Then inequality (36) is satisfied with $n_{\Theta}=\frac{\left(e^{\frac{\sqrt{6}}{4}}-1\right)^{3}}{8 \sqrt{\pi}}>0$ and $\Theta \approx 2.901034837$. It follows that

$$
M_{f, \mathcal{B}}=\frac{\Theta n_{\mathcal{B}}}{1-\left(L_{1} \Psi_{1}(T, \alpha)+L_{2} \Psi_{1}(T, q+\alpha)\right)} \approx 0.1683734132>0 .
$$

Therefore by Theorem 4.12 problem (45) is both $\mathbb{U} \mathbb{H} \mathbb{R}$ and $\mathbb{G} U \mathbb{H} \mathbb{R}$ stable on $[0,3 / 2]$.

(iii) Consider the function

$$
\begin{aligned}
& f\left(\varsigma, x(\varsigma / 3), \mathcal{I}_{0^{+}}^{\sqrt{\pi} ; e^{\frac{\sqrt{\varsigma}}{2}}} x(\varsigma / 4)\right) \\
& \quad=\frac{e^{-3 \varsigma}}{\varsigma+1}+\frac{3}{2 \varsigma+5} \cdot \tan ^{-1}|x(\varsigma / 3)|+\frac{2}{(2 \varsigma+3)^{2}} \cdot \frac{\left|\mathcal{I}_{0^{+}}^{\sqrt{\pi} ; e^{\frac{\sqrt{\varsigma}}{2}}} x(\varsigma / 4)\right|}{2+\left|\mathcal{I}_{0^{+}}^{\sqrt{\pi} ; e^{\frac{\sqrt{\varsigma}}{2}}} x(\varsigma / 4)\right|} .
\end{aligned}
$$

with $\theta=1 / 3$ and $\varepsilon=1 / 4$. For $x_{i}, y_{i} \in \mathbb{R}, i=1,2$, and $\varsigma \in[0,3 / 2]$, we can find that

$$
\left|f\left(\varsigma, x_{1}, y_{1}\right)-f\left(\varsigma, x_{2}, y_{2}\right)\right| \leq \frac{3}{5}\left|x_{1}-x_{2}\right|+\frac{1}{9}\left|y_{1}-y_{2}\right| .
$$

This means that assumption $\left(H_{1}\right)$ is satisfied with $L_{1}=3 / 5$ and $L_{2}=1 / 9$. We have

$$
\Lambda\left(L_{1}, L_{2}\right)-L_{1} \Psi_{1}(T, \alpha)-L_{2} \Psi_{1}(T, q+\alpha) \approx 0.5034171857<1
$$

and

$$
|f(\varsigma, x, y)| \leq \frac{e^{-3 \varsigma}}{\varsigma+1}+\frac{3 \pi}{2(2 \varsigma+5)}+\frac{1}{(2 \varsigma+3)^{2}},
$$

which satisfy assumption $\left(H_{3}\right)$ and (27), respectively. Applying Theorem 3.6, we find a solution for the $\psi$-Hilfer FBVP describing the thermostat control model (45) on [0,3/2]. Moreover, we can also calculate that

$$
M_{f}=\frac{\Lambda(1,0)}{1-\left(L_{1} \Psi_{1}(T, \alpha)+L_{2} \Psi_{1}(T, q+\alpha)\right)} \approx 1.341638979>0
$$


Therefore by Theorem 4.10 problem (45) is both $\mathbb{U H}$ and $\mathbb{G U H}$ stable on [0,3/2]. In addition, if we set $\mathcal{B}(\varsigma)=(\psi(\varsigma)-\psi(0))^{3}$, then by Proposition $2.5(\mathrm{i})$ we easily compute that

$$
\mathcal{I}_{0^{+}}^{\alpha ; \psi} \mathcal{B}(\varsigma)=\frac{\Gamma(4)}{\Gamma(4+\alpha)}(\psi(\varsigma)-\psi(0))^{3+\alpha} \Theta(\varsigma) \leq \frac{64\left(e^{\frac{\sqrt{6}}{4}}-1\right)^{\frac{9}{2}}}{315 \sqrt{\pi}} \Theta(\varsigma)
$$

Then inequality (36) is satisfied with $n_{\Theta}=\frac{64\left(e^{\frac{\sqrt{6}}{4}}-1\right)^{\frac{9}{2}}}{315 \sqrt{\pi}}>0$ and $\Theta \approx 2.901034837$. It follows that

$$
M_{f, \mathcal{B}}=\frac{\Theta n_{\mathcal{B}}}{1-\left(L_{1} \Psi_{1}(T, \alpha)+L_{2} \Psi_{1}(T, q+\alpha)\right)} \approx 0.2424862463>0 .
$$

Therefore by Theorem 4.12 the $\psi$-Hilfer FBVP describing the thermostat control model (45) is both $\mathbb{U H} \mathbb{R}$ and $\mathbb{G} \mathbb{U H} \mathbb{R}$ stable on $[0,3 / 2]$.

\section{Conclusions}

Oriented by the recent trend that supports considering some well-known physical models in the frame of generalized fractional operators, we study the model of thermostat that controls the heating or cooling sources. The model is described using integro-differential equation in the context of the generalized $\psi$-Hilfer fractional operator. Unlike previous research works, we proved the existence and uniqueness of solutions for a generalized category of the $\psi$-Hilfer FBVP describing the thermostat control model. The fixed-point approaches due to Banach, Schaefer, and Krasnoselskii are used to establish the relevant results. Different kinds of the Ulam stability, such as $\mathbb{U H}, \mathbb{G} \mathbb{U H}$, $\mathbb{U H} \mathbb{R}$, and $\mathbb{G} \mathbb{U H} \mathbb{R}$ stability are also investigated. Moreover, the results are well confirmed by several numerical examples. Note that we can continue such a research by extending it to coupled systems of fractional thermostat BVPs in terms of newly defined fractional operators with nonsingular kernels. Also, to obtain the exact solutions of such a coupled system, there are different numerical algorithms, which we can implement on these generalized fractional models in the next studies.

\section{Acknowledgements}

The third author would like to thank Prince Sultan University for supporting this work. The fourth and fifth authors were supported by Azarbaijan Shahid Madani University. The authors express their gratitude to anonymous referees for their helpful suggestions which improved the final version of this paper.

Funding

Not applicable.

\section{Availability of data and materials}

Data sharing not applicable to this paper as no datasets were generated or analyzed during the current study.

Ethics approval and consent to participate

Not applicable.

Competing interests

The authors declare that they have no competing interests.

Consent for publication

Not applicable.

Authors' contributions

The authors declare that the study was realized in collaboration with equal responsibility. All authors read and approved the final manuscript. 


\section{Author details}

${ }^{1}$ Department of Mathematics, Faculty of Science, Burapha University, Chonburi, 20131, Thailand. ${ }^{2}$ Department of Applied Statistics, Faculty of Applied Science, King Mongkut's University of Technology North Bangkok, Bangkok, 10800, Thailand. ${ }^{3}$ Department of Mathematics and General Sciences, Prince Sultan University, Riyadh, 11586, Saudi Arabia. ${ }^{4}$ Department of Mathematics, Azarbaijan Shahid Madani University, Tabriz, Iran. ${ }^{5}$ Department of Medical Research, China Medical University Hospital, China Medical University, Taichung, Taiwan.

\section{Publisher's Note}

Springer Nature remains neutral with regard to jurisdictional claims in published maps and institutional affiliations.

Received: 26 February 2021 Accepted: 30 March 2021 Published online: 09 April 2021

\section{References}

1. Miller, K.S., Ross, B.: An Introduction to the Fractional Calculus and Fractional Differential Equations. Wiley, New York (1993)

2. Podlubny, |: Fractional Differential Equations. Academic Press, San Diego (1999)

3. Yang, A.M., Han, Y., Zhang, Y.Z., Wang, L.T., Zhang, D., Yang, X.J.: On nonlocal fractional Volterra integro-differential equations in fractional steady heat transfer. Therm. Sci. 20(3), 789-793 (2016). https://doi.org/10.2298/TSCI16S3789Y

4. Tarasov, V.E.: Fractional integro-differential equations for electromagnetic waves in dielectric media. Theor. Math. Phys. 158, 355-359 (2009). https://doi.org/10.1007/s11232-009-0029-z

5. Adiguzel, R.S., Aksoy, U., Karapinar, E., Erhan, I.M.: On the solution of a boundary value problem associated with a fractional differential equation. Math. Methods Appl. Sci. (2010). https://doi.org/10.1002/mma.6652

6. Afshari, H., Kalantari, S., Karapinar, E.: Solution of fractional differential equations via coupled fixed point. Electron. J. Differ. Equ. 15(286), 1 (2015). http://ejde.math.txstate.edu

7. Jajarmi, A., Baleanu, D.: On the fractional optimal control problems with a general derivative operator. Asian J. Control 23(2), 1062-1071 (2021). https://doi.org/10.1002/asjc.2282

8. Jajarmi, A., Baleanu, D.: A new iterative method for the numerical solution of high-order nonlinear fractional boundary value problems. Front. Phys. 8, 220 (2020). https://doi.org/10.3389/fphy.2020.00220

9. Karapinar, E., Fulga, A., Rashid, M., Shahid, L., Aydi, H.: Large contractions on quasi-metric spaces with an application to nonlinear fractional differential equations. Mathematics 7(5), 444 (2019). https://doi.org/10.3390/math7050444

10. Sajjadi, S.S., Baleanu, D., Jajarmi, A., Pirouz, H.M.: A new adaptive synchronization and hyperchaos control of a biological snap oscillator. Chaos Solitons Fractals 138, 109919 (2020). https://doi.org/10.1016/j.chaos.2020.109919

11. Baleanu, D., Jajarmi, A., Mohammadi, H., Rezapour, S.: A new study on the mathematical modelling of human liver with Caputo-Fabrizio fractional derivative. Chaos Solitons Fractals 134, 109705 (2020). https://doi.org/10.1016/j.chaos.2020.109705

12. Baleanu, D., Ghanbari, B., Asad, J.H., Jajarmi, A., Pirouz, H.M.: Planar system-masses in an equilateral triangle: numerical study within fractional calculus. Comput. Model. Eng. Sci. 124(3), 953-968 (2020). https://doi.org/10.32604/cmes.2020.010236

13. Mohammadi, H., Kumar, S., Rezapour, S., Etemad, S.: A theoretical study of the Caputo-Fabrizio fractional modeling for hearing loss due to Mumps virus with optimal control. Chaos Solitons Fractals 144, 110668 (2021). https://doi.org/10.1016/j.chaos.2021.110668

14. Alqahtani, B., Aydi, H., Karapinar, E., Rakocevic, V.: A solution for Volterra fractional integral equations by hybrid contractions. Mathematics 7(8), 694 (2019). https://doi.org/10.3390/math7080694

15. Tuan, N.H., Mohammadi, H., Rezapour, S.: A mathematical model for COVID-19 transmission by using the Caputo fractional derivative. Chaos Solitons Fractals 140, 110107 (2020). https://doi.org/10.1016/j.chaos.2020.110107

16. Baleanu, D., Aydogan, S.M., Mohammadi, H., Rezapour, S.: On modelling of epidemic childhood diseases with the Caputo-Fabrizio derivative by using the Laplace Adomian decomposition method. Alex. Eng. J. 59(5), 3029-3039 (2020). https://doi.org/10.1016/j.aej.2020.05.007

17. Rezapour, S., Ntouyas, S.K., Iqbal, M.Q., Hussain, A., Etemad, S., Tariboon, J.: An analytical survey on the solutions of the generalized double-order $\phi$-integrodifferential equation. J. Funct. Spaces 2021, Article ID 6667757 (2021). https://doi.org/10.1155/2021/6667757

18. Baleanu, D., Etemad, S., Rezapour, S.: On a fractional hybrid integro-differential equation with mixed hybrid integral boundary value conditions by using three operators. Alex. Eng. J. 59(5), 3019-3027 (2020). https://doi.org/10.1016/j.aej.2020.04.053

19. Infante, G., Webb, J.: Loss of positivity in a nonlinear scalar heat equation. Nonlinear Differ. Equ. Appl. 13, 249-261 (2006). https://doi.org/10.1007/s00030-005-0039-y

20. Nieto, J.J., Pimentel, J.: Positive solutions of a fractional thermostat model. Bound. Value Probl. 2013, 5 (2013). https://doi.org/10.1186/1687-2770-2013-5

21. Aydi, H., Jleli, M., Samet, B.: On positive solutions for a fractional thermostat model with a convex-concave source term via $\Psi$-Caputo fractional derivative. Mediterr. J. Math. 2020, 17 (2020). https://doi.org/10.1007/s00009-019-1450-7

22. Caballero, J., Harjani, J., Sadarangani, K.: Uniqueness of solutions for a fractional thermostat model. Carpath. J. Math. 36(2), 223-228 (2020). https://www.jstor.org/stable/26918248

23. Baleanu, D., Etemad, S., Rezapour, S.: A hybrid Caputo fractional modeling for thermostat with hybrid boundary value conditions. Bound. Value Probl. 2020, 64 (2020). https://doi.org/10.1186/s13661-020-01361-0

24. Garai, H., Dey, L.K., Chanda, A.: Positive solutions to a fractional thermostat model in Banach spaces via fixed point results. J. Fixed Point Theory Appl. 20, 106 (2018). https://doi.org/10.1007/s11784-018-0584-8

25. Hao, X., Zhang, L.: Positive solutions of a fractional thermostat model with a parameter. Symmetry 11(1), 122 (2019). https://doi.org/10.3390/sym11010122

26. Hao, X., Sun, H., Liu, L.: Existence results for fractional integral boundary value problem involving fractional derivatives on an infinite interval. Math. Methods Appl. Sci. 41(16), 6984-6996 (2018). https://doi.org/10.1002/mma.5210 
27. Vanterler da C. Sousa, J., Capelas de Oliveira, E.: On the Ulam-Hyers-Rassias stability for nonlinear fractional differential equations using the $\psi$-Hilfer operator. J. Fixed Point Theory Appl. 20, 96 (2018). https://doi.org/10.1007/s11784-018-0587-5

28. Harikrishman, S., Elsayed, E., Kanagarajan, K.: Existence and uniqueness results for fractional pantograph equations involving $\psi$-Hilfer fractional derivative. Dyn. Contin. Discrete Impuls. Syst., Ser. A Math. Anal. 25, 319-328 (2018) http://www.watam.org

29. Ahmed, I., Kuman, P., Shah, K., Borisut, P., Sitthithakerngkiet, K., Demba, M.A.: Stability results for implicit fractional pantograph differential equations via $\phi$-Hilfer fractional derivative with a nonlocal Riemann-Liouville fractional integral condition. Mathematics 8(1), 94 (2018). https://doi.org/10.3390/math8010094

30. Ben-Chikh, S., Amara, A., Etemad, S., Rezapour, S.: On Ulam-Hyers-Rassias stability of a generalized Caputo type multi-order boundary value problem with four-point mixed integro-derivative conditions. Adv. Differ. Equ. 2020, 680 (2020). https://doi.org/10.1186/s13662-020-03139-1

31. Boutiara, A., Etemad, S., Hussain, A., Rezapour, S.: The generalized U-H and U-H stability and existence analysis of a coupled hybrid system of integro-differential IVPs involving $\phi$-Caputo fractional operators. Adv. Differ. Equ. 2021, Article ID 95 (2021). https://doi.org/10.1186/s13662-021-03253-8

32. Vanterler da C. Sousa, J., Capelas de Oliveira, E.: Ulam-Hyers stability of a nonlinear fractional Volterra integro-differential equation. Appl. Math. Lett. 81, 50-56 (2018). https://doi.org/10.1016/j.aml.2018.01.016

33. Vanterler da C. Sousa, J., Rodrigues, F.G., Capelas de Oliveira, E.: Stability of the fractionalVolterra integro-differential equation by means of $\psi$-Hilfer operator. Math. Methods Appl. Sci. 42(9), 3033-3043 (2019). https://doi.org/10.1002/mma.5563

34. Kilbas, A.A., Srivastava, H.M., Trujillo, J.J.: Theory and Applications of Fractional Differential Equations. Elsevier, Amsterdam (2006)

35. Vanterler da C. Sousa, J., Capelas de Oliveira, E.: On the $\psi$-Hilfer fractional derivative. Commun. Nonlinear Sci. Numer. Simul. 60, 72-91 (2018). https://doi.org/10.1016/j.cnsns.2018.01.005

36. Granas, A., Dugundji, J.: Fixed Point Theory. Springer, New York (2003)

37. Krasnoselskii, M.A.: Two remarks on the method of successive approximations. Usp. Mat. Nauk 10, 123-127 (1955)

38. Rus, I.A.: Ulam stabilities of ordinary differential equations in a Banach space. Carpath. J. Math. 26(1), 103-107 (2010) https://www.jstor.org/stable/43999438

\section{Submit your manuscript to a SpringerOpen ${ }^{\circ}$ journal and benefit from:}

- Convenient online submission

- Rigorous peer review

- Open access: articles freely available online

- High visibility within the field

- Retaining the copyright to your article

Submit your next manuscript at $\boldsymbol{\nabla}$ springeropen.com 